\title{
An Analytical Approach for Performance Evaluation of BICM Transmission over Nakagami-m Fading Channels
}

\author{
Alireza Kenarsari-Anhari and Lutz Lampe \\ University of British Columbia, Vancouver, BC, Canada \\ \{alirezak, Lampe\}@ece.ubc.ca
}

\begin{abstract}
Bit-interleaved coded modulation (BICM) has established itself as a quasi-standard for bandwidth- and power-efficient wireless communication. In this paper, we present an analytical approach to evaluate the performance of BICM transmission over frequency-flat fading additive white Gaussian noise channels. The statistic of the fading envelope is modeled as Nakagami- $m$ distributed, which spans a wide range of practical multipath fading scenarios through adjustment of the $m$-parameter. For this setup, we derive approximations for the bit-error rate (BER) and cutoff rate of BICM. Different from previously proposed methods, our analysis is valid for general quadrature amplitude modulation and phase shift keying signal constellations and arbitrary bit-to-symbol mapping rules, and it results in simple closed-form expressions. The key idea is to use well-chosen subsets of signal points to approximate the probability density function of reliability metrics used for decoding. This approximation is accurate for signal-to-noise ratio regions of interest for BICM systems with moderate coding complexity such as, e.g., convolutional coded BICM systems. Based on this approximation we also derive an asymptotic BER expression, which reveals the diversity order and coding gain of BICM. The usefulness of the proposed analytical approach is validated through numerical and simulation results for a number of BICM transmission examples.
\end{abstract}

\section{Index Terms}

Bit-interleaved coded modulation (BICM), performance analysis, cutoff rate, saddlepoint approximation, fading channels.

Part of this work has been presented at the 2009 IEEE International Conference on Communications (ICC), Dresden, Germany. 


\section{INTRODUCTION}

Bit-interleaved coded modulation (BICM) introduced in [1] and further generalized in [2] has established itself as the most popular scheme for spectrally efficient coded transmission. BICM connects a binary encoder to a non-binary modulator and achieves nearly optimal performance in terms of, e.g., constellation-constrained channel capacity [2]. The most noticeable advantage of BICM is its simplicity and flexibility, as a single binary code can be used along with several modulations without further adaptations. BICM was originally designed as a superior alternative to trellis coded modulation [3] in fading channels [1]. However, its flexibility and ease of implementation also make BICM an attractive scheme for transmission over nonfading channels [2], [4].

Different bounds for the bit error-rate (BER) of BICM have been derived in previous works. The Bhattacharyya union bound was found to be quite loose but a true upper bound for arbitrary constellation labeling rules [2]. A refined technique, which was also developed in [2] and referred to as BICM Expurgated bound (BICM-EB), provides tighter results but is more complex to compute and limited to the Gray labeling. Also, [5] pointed out that the BICM-EB is not an upper bound but rather an error-rate approximation. A generalized version of the BICM-EB has been proposed in [6] which considers finite-length interleaving, but again is limited to the Gray labeling and numerically more complex to compute than the bounds given in [2]. The authors of [4], [7] presented two new approximations, namely, the Gaussian and saddlepoint approximations. The former is based on the Gaussian approximation of the tail of the probability density function (PDF) of the bitwise loglikelihood ratio (LLR) while the latter is an application of the saddlepoint approximation technique known from statistics [8]. Both approximations are applicable to arbitrary labeling rules but rely on numerical integration using various Gauss quadrature rules for computing the cumulant generating function of the LLRs [7].

The need for numerical integration renders the aforementioned approximations complex to compute and rather hard to use as a design tool. Recently, [9] devised an algorithmic approach to compute the PDF of LLRs for the nonfading additive white Gaussian noise (AWGN) channel applicable to arbitrary labeling rules. However, this method results in PDF expressions which are not simple and 
thus, evaluation of performance expressions, as for example BER, based on these PDF expressions again invokes numerical techniques. These problems have been overcome in the follow-up work [10], where a closed-form expression for the PDF for square quadrature amplitude modulation (QAM) with Gray labeling is obtained, and then further simplified using a Gaussian approximation. In [11] (cf. also [12]), closed-form PDF expressions are derived for BICM transmission over Nakagami- $m$ fading channels with integer $m$, which are applied for BER approximation using the saddlepoint technique. Again, the approach is restricted to QAM with Gray labeling. During the revision of this paper, we became aware of [13], which derives a BER approximation for Nakagami-m fading channels using a "large-SNR" analysis. Also this analysis requires Gray labeling.

In this paper we take a fresh look at the performance evaluation of BICM transmission over frequency-flat fading AWGN channels. More specifically, we consider fading according to the Nakagami$m$ distribution with general real-valued $m$, which through adjustment of the $m$ parameter spans the widest range of "fading figure" among the well-known fading distributions [14], and includes the popular Rayleigh fading and nonfading channels as special cases. Our results are performance approximations, which become accurate for signal-to-noise power ratio (SNR) regions where the BER union bound converges to the true error rate.

The main contributions of this work can be summarized as follows. (i) A new closed-form approximation for the PDF of bitwise reliability metrics when transmitting over the nonfading AWGN channel is derived. The key idea is to use well-chosen subsets of nearest-neighbor signal points for LLR approximation. The resulting PDF expression is valid for arbitrary modulation and labeling rules. Different from [9], the simplicity of our novel PDF approximation enables the expression of pertinent BICM performance parameters in closed form. (ii) Towards this end, we derive the Laplace transform of the newly found PDF expression for general Nakagami- $m$ fading AWGN channels. Using this result together with the saddlepoint approximation, closed-form expressions for the pairwise error probability (PEP) between codewords are obtained. The BER for BICM with linear codes is then easily upper bounded in terms of these PEP expressions. (iii) In addition to BER, also the cutoff rate for BICM, which has been used as a parameter to predict the performance of BICM with moderate- 
complexity coding schemes [2], is written in terms of the mentioned Laplace transform and thus can be approximated in closed form. (iv) Based on the new PDF approximation we also derive asymptotic BER expressions as the SNR goes to infinity. It is shown that for the nonfading channel the BER is closely approximated by the BER expression for an equivalent binary transmission scaled by a constant which is a function of the minimum Hamming distance $d_{\mathrm{H}, \mathrm{min}}$ of the code and the labeling rule. For the case of Nakagami- $m$ fading it is shown that the diversity order is the product of $m$ and $d_{\mathrm{H}, \mathrm{min}}$. Furthermore, the asymptotic coding gain is shown to depend on a parameter which is a generalization of the harmonic mean presented in [2]. (v) A set of numerical and simulation results for BICM with convolutional codes is presented, which provides evidence of the tightness of the proposed approximations, including the asymptotic BER expressions.

The remainder of this paper is organized as follows. In Section II the BICM transmission model is introduced, and the formulation of BER union bound, saddlepoint approximation for PEP, and cutoff rate for BICM is briefly reviewed. The novel closed-form approximation for the PDF of LLRs and its Laplace transform are derived in Section III. The detailed solutions of a number of integrals encountered during the derivations are relegated to the appendix. The asymptotic BER analysis for large SNR is provided in Section IV. Comparisons between the proposed analytical approximations and simulation results are given in Section V. Finally, conclusions are offered in Section VI.

\section{PRELIMINARIES}

In this section, we first introduce the BICM transmission model. Then, we briefly review error-rate approximations for such systems using union bounding and saddlepoint approximation techniques. Furthermore, we present an expression for the BICM cutoff rate.

\section{A. System Model}

Fig. 1 shows the block diagram of the equivalent baseband BICM transmission system.

1) Transmitter: The BICM codeword $\boldsymbol{x}=\left[x_{1}, x_{2}, \ldots, x_{L}\right] \in \mathbb{C}$ comprises $L$ complex valued symbols and is obtained by first interleaving $(\pi)$ the output of a binary encoder $c=\left[c_{1}, c_{2}, \ldots, c_{N}\right] \in \mathbb{F}_{2}^{N}$

into $c^{\pi}=\left[c_{1}^{\pi}, c_{2}^{\pi}, \ldots, c_{N}^{\pi}\right] \in \mathbb{F}_{2}^{N}$ and a subsequent mapping $\mu:\{0,1\}^{r} \rightarrow \mathcal{X}$ of each $r \triangleq \log _{2}(M)$ bits 
such that $x_{i}=\mu\left(\left[c_{(i-1) r+1}^{\pi}, c_{(i-1) r+2}^{\pi}, \ldots, c_{i r}^{\pi}\right]\right)$. While the PDF approximation presented in Section III is applicable to arbitrary signal constellations, for better readability and practical relevance we assume that $\mathcal{X}$ is an $M$-ary QAM or phase-shift keying (PSK) constellation with average unit symbol energy. As usual, we assume that coding and mapping results in a uniform distribution of signal points. When presenting numerical results in Sections III and V, we consider set partitioning labeling (SPL), semi set partitioning labeling (SSPL), modified set partitioning labeling (MSPL), and mixed labeling (ML) (cf. [2], [15], [16]) in addition to popular (binary reflected) Gray labeling (GL) [17] for QAM and PSK signal constellations.

2) Channel: We consider BICM transmission over AWGN channels. Making the usual assumptions about synchronization, filtering, sampling, and channel-phase compensation in a coherent receiver, the equivalent baseband discrete-time transmission model can be written as

$$
y_{i}=\sqrt{\bar{\gamma}} h_{i} x_{i}+z_{i}
$$

where $y_{i} \in \mathbb{C}$ is the received sample, $h_{i} \in \mathbb{R}$ denotes the fading gain, $z_{i} \in \mathbb{C}$ is the additive noise sample at discrete-time $i$. The noise samples are assumed to be independent and identically distributed (i.i.d.) according to a zero-mean complex Gaussian distribution. We further assume that interleaving effectively renders the fading coefficients $h_{i}$ i.i.d. random variables. Applying normalization such that $h_{i}$ and $z_{i}$ have average power one, $\bar{\gamma}$ represents the average SNR. The instantaneous SNR is given by

$$
\gamma_{i}=\bar{\gamma} h_{i}^{2}
$$

To make matters concrete we consider the widely used Nakagami- $m$ distribution to model multipath fading. Adjustment of the fading parameter $m \geq 1 / 2$ renders this distribution very flexible. It includes Rayleigh fading $(m=1)$ and nonfading AWGN $(m \rightarrow \infty)$ channels as special cases [14, Ch. 2.2.14]. The corresponding distribution of the SNR (2) reads [14] $(\Gamma(\cdot)$ denotes the Gamma function)

$$
f_{\gamma \mid \bar{\gamma}, m}(\gamma)=\frac{m^{m} \gamma^{m-1}}{\bar{\gamma}^{m} \Gamma(m)} \exp \left(-\frac{m \gamma}{\bar{\gamma}}\right) \text {. }
$$


3) Receiver: At the receiver, the demapper $\left(\mu^{-1}\right.$ in Fig. 1) produces $r$ bitwise reliability metrics $\Lambda^{\pi}$ per symbol. The $\Lambda^{\pi}$ are deinterleaved into $\Lambda$, which are then input to the decoder for the binary code. The bit metric for the $j$ th bit of the $i$ th symbol has the form

$$
\Lambda=-\min _{a \in \mathcal{X}_{j, 1}}\left(\left\|y_{i}-\sqrt{\bar{\gamma}} h_{i} a\right\|^{2}\right)+\min _{a \in \mathcal{X}_{j, 0}}\left(\left\|y_{i}-\sqrt{\bar{\gamma}} h_{i} a\right\|^{2}\right)
$$

where $\mathcal{X}_{j, b}$ is the set of symbols with the $j$ th bit in the binary label fixed to $b$. We note that (4) is the so-called max-log simplification of the LLR, which is known to provide practically maximumlikelihood decoding performance, cf. e.g. [2], [18]. Therefore we adopt this simple metric expression (cf. also [9]-[11]). In slight abuse of terminology ${ }^{1}$, we will refer to $\Lambda$ using (4) as LLR in the following.

\section{B. Error-Rate Approximation Using Union Bounding and Saddlepoint Approximation}

The transmission channel between encoder output $c$ and decoder input $\Lambda$ can be considered as an equivalent binary-input output-symmetric (BIOS) channel [7], which is known as equivalent BICM channel (see Fig. 1). ${ }^{2}$ Assuming maximum-likelihood decoding, the error-rate of linear codes transmitted over BIOS channels is well approximated by the union bound in the region above the cutoff rate [19]. For example, the BER union bound for a convolutional code of rate $R_{c}=k_{c} / n_{c}$ is given by

$$
P_{b} \leq \frac{1}{k_{c}} \sum_{d_{\mathrm{H}}=d_{\mathrm{H}, \text { min }}}^{\infty} w_{d_{\mathrm{H}}} \operatorname{PEP}\left(d_{\mathrm{H}} \mid \bar{\gamma}, m\right),
$$

where $w_{d_{\mathrm{H}}}$ denotes total input weight of error events at Hamming distance $d_{\mathrm{H}}, d_{\mathrm{H} \text {,min }}$ denotes the free distance of the convolutional code, and $\operatorname{PEP}\left(d_{\mathrm{H}} \mid \bar{\gamma}, m\right)$ is the PEP corresponding to an error event with Hamming weight $d_{\mathrm{H}}$. For clarity, we made the dependency of $\operatorname{PEP}\left(d_{\mathrm{H}} \mid \bar{\gamma}, m\right)$ on average SNR $\bar{\gamma}$ and fading parameter $m$ explicit.

\footnotetext{
${ }^{1}$ Strictly speaking, the term LLR is reserved for metrics which are based on the likelihood function.

${ }^{2}$ In [2], it has been shown that for BICM systems with signal constellation $\mathcal{X}$ and/or labeling $\mu$ which do not preserve the symmetry of the output an equivalent BIOS channel could be considered by switching between the labeling $\mu$ and its complement $\bar{\mu}$ with probability of $1 / 2$. Symmetrization does not affect the performance under the common hypothesis of uniform encoder outputs.
} 
For BIOS channels, the PEP can be considered as the tail probability of a random variable generated by summing $d_{\mathrm{H}}$ i.i.d. LLRs $\Lambda_{1}, \ldots, \Lambda_{d_{\mathrm{H}}}$. More specifically, choosing the all-one codeword as reference codeword, we have

$$
\operatorname{PEP}\left(d_{\mathrm{H}} \mid \bar{\gamma}, m\right)=\operatorname{Pr}\left(\Delta_{d_{\mathrm{H}}} \triangleq \sum_{i=1}^{d_{\mathrm{H}}} \Lambda_{i}<0 \mid \bar{\gamma}, m\right) .
$$

A common approach for computing such a probability is through the use of the Laplace transform $\Phi_{\Lambda \mid \bar{\gamma}, m}(s)$ of the PDF of $\Lambda$. That is [20]

$$
\operatorname{Pr}\left(\Delta_{d_{\mathrm{H}}}<0 \mid \bar{\gamma}, m\right)=\frac{1}{2 \pi \mathrm{j}} \int_{\alpha-\mathrm{j} \infty}^{\alpha+\mathrm{j} \infty}\left[\Phi_{\Lambda \mid \bar{\gamma}, m}(s)\right]^{d_{\mathrm{H}}} \frac{d s}{s},
$$

where $\mathrm{j} \triangleq \sqrt{-1}$ and $\alpha \in \mathbb{R}, 0<\alpha<\alpha_{\max }$, is chosen in the region of convergence of the integral. The computation of (7) itself is often not straightforward and invokes the use of numerical methods [20]. For this reason [20] has proposed a few bounds and estimations, among which the saddlepoint approximation has recently attracted considerable interest due to its simple form and accuracy [4], [7]. Approximation of (7) using the saddlepoint technique results in ${ }^{3}$ [7], [20]

$$
\operatorname{Pr}\left(\Delta_{d_{\mathrm{H}}}<0 \mid \bar{\gamma}, m\right) \approx \frac{1}{\hat{s} \sqrt{2 \pi d_{\mathrm{H}} \Phi_{\Lambda \mid \bar{\gamma}, m}^{\prime \prime}(\hat{s})}}\left(\Phi_{\Lambda \mid \bar{\gamma}, m}(\hat{s})\right)^{\left(d_{\mathrm{H}}+0.5\right)},
$$

where $\Phi_{\Lambda \mid \bar{\gamma}, m}^{\prime \prime}(s)$ denotes the second-order derivative of $\Phi_{\Lambda \mid \bar{\gamma}, m}(s)$ and $\hat{s} \in \mathbb{R}^{+}, 0<\hat{s}<\alpha_{\max }$, is the saddlepoint defined through the first-order derivative as

$$
\Phi_{\Lambda \mid \bar{\gamma}, m}^{\prime}(\hat{s})=0
$$

While $\hat{s}=1 / 2$ for BIOS channels with maximum-likelihood decoding, a slightly different saddlepoint may be found for the max-log approximated LLR in (4) [12].

\section{Cutoff Rate}

From (7) we can write the Chernoff bound for the PEP between two codewords $c$ and $c^{\prime}$ as ( $\oplus$ denotes addition in $\mathbb{F}_{2}$ )

$$
\operatorname{Pr}\left(\boldsymbol{c} \rightarrow \boldsymbol{c}^{\prime}\right) \leq \min _{0<s<\alpha_{\max }}\left(\prod_{i=1}^{N}\left(\Phi_{\Lambda \mid \bar{\gamma}, m}(s)\right)^{c_{i} \oplus c_{i}^{\prime}}\right),
$$

${ }^{3}$ Note that in [7] the saddlepoint approximation is written in terms of the cumulant transform $\kappa(s)$ and that [7, Eq. (12] is identical to $(8)$ since $\kappa(s)=\log [\Phi(-s)]$ and $\Phi^{\prime}(\hat{s})=0$. 
and thus express the cutoff rate as [21]

$$
R_{0}=-r \log _{2}\left[\min _{0<s<\alpha_{\max }}\left(\sum_{c \in\{0,1\}} \sum_{c^{\prime} \in\{0,1\}} \operatorname{Pr}(c) \operatorname{Pr}\left(c^{\prime}\right)\left(\Phi_{\Lambda \mid \bar{\gamma}, m}(s)\right)^{c \oplus c^{\prime}}\right)\right] .
$$

The factor $r$ in (11) renders the unit of $R_{0}$ bit per transmission symbol. With the assumption of uniformly distributed coded bits, (11) can be rewritten as

$$
R_{0}=r\left(1-\log _{2}\left[1+\Phi_{\Lambda \mid \bar{\gamma}, m}(\hat{s})\right]\right)
$$

with $\hat{s}$ from (9).

We observe that the evaluation of the error-rate approximation via (8) and the cutoff rate (12) hinge on expressions for the Laplace transform $\Phi_{\Lambda \mid \bar{\gamma}, m}(s)$ for $s \in \mathbb{R}^{+}$. The derivation of closed-form approximations for $\Phi_{\Lambda \mid \bar{\gamma}, m}(s)$ is considered in the next section.

\section{Approximation fOR the PDF of LLRs And its LAplace Transform}

In this section, we first derive an approximation for the PDF of the LLRs defined in (4) assuming transmission over the nonfading AWGN channel (i.e., $m \rightarrow \infty$ ). Using this approximation, we then derive closed-form expressions for $\Phi_{\Lambda \mid \bar{\gamma}, m}(s)$ for $s \in \mathbb{R}^{+}$and arbitrary $m$.

\section{A. Approximation for the PDF of LLRs}

We denote the PDF of LLRs (4) for the nonfading channel with $c=b$ being transmitted by $f_{\Lambda \mid c=b, \gamma}(\lambda)$ and the complement of $b$ by $\bar{b}=b \oplus 1$. Since the channel is BIOS, the symmetry property

$$
f_{\Lambda \mid c=b, \gamma}(\lambda)=f_{\Lambda \mid c=\bar{b}, \gamma}(-\lambda)
$$

holds, and thus we consider the transmission of $c=1$ without loss of generality.

The PDF of LLRs can be considered as a weighted sum of PDFs $f_{\Lambda \mid j, x, \gamma}(\lambda)$ conditioned on the bit position $1 \leq j \leq r$ and the transmitted symbol $x \in \mathcal{X}_{j, 1}$ :

$$
f_{\Lambda \mid c=1, \gamma}(\lambda)=\frac{1}{r} \sum_{j=1}^{r} \sum_{x \in \mathcal{X}_{j, 1}} \operatorname{Pr}(x \mid c=1, j) f_{\Lambda \mid j, x, \gamma}(\lambda)=\frac{2}{r M} \sum_{j=1}^{r} \sum_{x \in \mathcal{X}_{j, 1}} f_{\Lambda \mid j, x, \gamma}(\lambda),
$$

where the second step follows from the assumption of equiprobable modulator inputs. Hence, the task is to find expressions for $f_{\Lambda \mid j, x, \gamma}(\lambda)$ for every $1 \leq j \leq r$ and $x \in \mathcal{X}_{j, 1}$. This requires consideration 
of all signal points in the constellation and, depending on the type of modulation and labeling, may not lead to a closed-form result.

To motivate our approach, consider a transmitted codeword $c$ and a given competitive codeword $\boldsymbol{c}^{\prime}$ with Hamming distance of $d_{\mathrm{H}}$ from $\boldsymbol{c}$. Assuming that the $d_{\mathrm{H}}$ distinct bits of $\boldsymbol{c}$ are transmitted using $d_{\mathrm{H}}$ symbols and label positions $\boldsymbol{j}_{\boldsymbol{c}}=\left[j_{1}, j_{2}, \ldots, j_{d_{\mathrm{H}}}\right]$, the corresponding bits of $\boldsymbol{c}^{\prime}$ could be transmitted using any sequence of the signal points in

$$
\mathcal{X}_{c^{\prime}} \triangleq\left\{\mathcal{X}_{j_{1}, c_{1}^{\prime}} \times \mathcal{X}_{j_{2}, c_{2}^{\prime}} \times \ldots \times \mathcal{X}_{j_{d_{\mathrm{H}}-1}, c_{d_{\mathrm{H}}-1}^{\prime}} \times \mathcal{X}_{j_{d_{\mathrm{H}}}, c_{d_{\mathrm{H}}}^{\prime}}\right\}
$$

Using the union upper bound over $\mathcal{X}_{c^{\prime}}$ results in the BICM Union Bound [2, Section IV.B]. In this case, $f_{\Lambda \mid j, x, \gamma}(\lambda)$ is approximated by considering all signal points in $\mathcal{X}_{j, \bar{b}}$ for $x \in \mathcal{X}_{j, b}$. Replacing $\mathcal{X}_{j, c^{\prime}}$ with a single, nearest-neighbor signal point leads to the BICM-EB [2, Section IV.C]. That is, the BICM-EB estimates $f_{\Lambda \mid j, x, \gamma}(\lambda)$ by considering only one member of $\mathcal{X}_{j, \bar{b}}$, which is not a valid simplification for non-Gray labeling rules due to the presence of multiple nearest neighbors [2], [6], [7]. Instead of these two extreme approaches, we propose to use the set of all nearest signal points in $\mathcal{X}_{j, \bar{b}}$ for a given $x \in \mathcal{X}_{j, b}$. That is, we define the set of nearest competitive signal points of $x$,

$$
\mathcal{A}_{j, x} \triangleq\left\{a \mid a \in \mathcal{X}_{j, \bar{b}},\|a-x\|=\min _{a^{\prime} \in \mathcal{X}_{j, \bar{b}}}\left\|a^{\prime}-x\right\| \triangleq d_{j, x}\right\},
$$

to approximate $f_{\Lambda \mid j, x, \gamma}(\lambda)$. We note that this corresponds to the approximation

$$
\Lambda \approx-\left(\left\|y_{i}-\sqrt{\bar{\gamma}} h_{i} x_{i}\right\|^{2}\right)+\min _{a \in \mathcal{A}_{j, x_{i}}}\left(\left\|y_{i}-\sqrt{\bar{\gamma}} h_{i} a\right\|^{2}\right)
$$

for the LLR in (4), which is expected to be accurate in the SNR range in which the minimum Euclidean distance events dominate and the BER union bound converges to the true error rate.

There are six non-equivalent formations for the sets of nearest competitive signal points $\mathcal{A}_{j, x}$ for QAM and PSK constellations. These are illustrated in Fig. 2. For each formation we determine the $\operatorname{PDF} f_{\Lambda \mid j, x, \gamma}(\lambda)$ from the corresponding cumulative density function

$$
F_{\Lambda \mid j, x, \gamma}(\lambda)=\operatorname{Pr}(z \in \mathbb{D}(\lambda \mid j, x, \gamma))
$$

where $\mathbb{D}(\lambda \mid j, x, \gamma)$ is the part of complex plane in which the LLR according to (16) is less than $\lambda$ (see Fig. 2 for $\lambda=0$ ). Thus, the PDF is expressed by (recall that the noise power is normalized to 
one in (1))

$$
f_{\Lambda \mid j, x, \gamma}(\lambda)=\frac{\mathrm{d}}{\mathrm{d} \lambda} \int_{\mathbb{D}(\lambda \mid j, x, \gamma)} \frac{1}{\pi} \exp \left(-\|z\|^{2}\right) d z
$$

whose closed-form solution for the $k$ th configuration from Fig. 2 is specified as $f_{\Lambda, k \mid \cdot, \gamma}(\lambda)$ in Table I. For the expressions in Table I we used the notations

$$
\mathcal{N}_{\mu, \sigma^{2}}(x) \triangleq \frac{1}{\sqrt{2 \pi \sigma^{2}}} \exp \left(-\frac{(x-\mu)^{2}}{2 \sigma^{2}}\right), \operatorname{erf}(x) \triangleq \frac{2}{\sqrt{\pi}} \int_{0}^{x} \exp \left(-t^{2}\right) d t, u(x) \triangleq\left\{\begin{array}{ll}
1, & x \geq 0 \\
0, & x<0
\end{array} .\right.
$$

Substituting $f_{\Lambda \mid j, x, \gamma}(\lambda)$ in (14) with the corresponding $f_{\Lambda, k \mid, \gamma}(\lambda)$ from Table I gives us the desired closed-form expression for $f_{\Lambda \mid c=1, \gamma}(\lambda)$. For a general $M$-ary QAM constellation we obtain

$$
f_{\Lambda \mid c=1, \gamma}^{\mathrm{QAM}}(\lambda)=\frac{2}{r M} \sum_{k=1}^{5} \sum_{l=1}^{q_{\max }} n_{k, l} f_{\Lambda, k \mid d_{l}, \gamma}(\lambda), \quad d_{l}=l d_{\min },
$$

where $n_{k, l}$ denotes the number of nearest competitive signal sets of type $k$ with Euclidean distance $d_{l}, d_{\text {min }}$ is the minimum Euclidean distance of the constellation, and

$$
q_{\max } \triangleq \max _{1 \leq j \leq r}\left\{\max _{a \in \mathcal{X}_{j, 1}}\left\{\max _{a^{\prime} \in \mathcal{A}_{j, a}}\left\{\frac{\left\|a-a^{\prime}\right\|}{d_{\min }}\right\}\right\}\right\} .
$$

Similarly, for an $M$-ary PSK constellation we find

$$
f_{\Lambda \mid c=1, \gamma}^{\mathrm{PSK}}(\lambda)=\frac{2}{r M} \sum_{l=1}^{\frac{M}{2}}\left[n_{1, l} f_{\Lambda, 1 \mid d_{l}, \gamma}(\lambda)+n_{6, l} f_{\Lambda, 6 \mid d_{l}, \theta_{l}, \gamma}(\lambda)\right], \quad\left\{\begin{array}{l}
d_{l}=\frac{\sin \left(\frac{\pi l}{M}\right)}{\sin \left(\frac{\pi}{M}\right)} d_{\min } \\
\theta_{l}=\pi-\frac{2 \pi l}{M} .
\end{array}\right.
$$

Numerical values for $n_{k, l}$ are summarized for some popular signal constellations and labelings in Table II. It should be noted that the expressions in (20) and (21) are much easier to evaluate than the PDF approximations in [9]. In particular, their computation does not require any numerical integration.

Fig. 3 shows a comparison of PDF histograms, obtained through Monte Carlo simulation, and the approximations (20) and (21) for different constellations, labelings, and SNRs. We observe that the proposed approximation is very accurate regardless of the constellation and type of labeling. Especially the negative tail of the PDF is represented faithfully, which is important when evaluating performance parameters. 


\section{B. Laplace Transform of the PDF Approximation}

We now apply (20) and (21) to obtain expressions for the Laplace transform $\Phi_{\Lambda \mid \bar{\gamma}, m}(s)$ which become closed form for $s \in \mathbb{R}^{+}$.

1) Nonfading Channel: First we consider the nonfading case, i.e., $m \rightarrow \infty$, for which $\gamma=\bar{\gamma}$. We denote the Laplace transform for this case by $\Phi_{\Lambda \mid \gamma}(s)$. Starting from (20) and (21), $\Phi_{\Lambda \mid \gamma}(s)$ is obtained as

$$
\Phi_{\Lambda \mid \gamma}(s)=\frac{2}{r M} \sum_{k=1}^{5} \sum_{l=1}^{q_{\max }} n_{k, l} \Phi_{\Lambda, k \mid d_{l}, \gamma}(s), \quad d_{l}=l d_{\min }
$$

for a general $M$-ary QAM constellation and as

$$
\Phi_{\Lambda \mid \gamma}(s)=\frac{2}{r M} \sum_{l=1}^{\frac{M}{2}}\left[n_{1, l} \Phi_{\Lambda, 1 \mid d_{l}, \gamma}(s)+n_{6, l} \Phi_{\Lambda, 6 \mid d_{l}, \theta_{l}, \gamma}(s)\right], \quad\left\{\begin{aligned}
d_{l} & =\frac{\sin \left(\frac{\pi l}{M}\right)}{\sin \left(\frac{\pi}{M}\right)} d_{\text {min }}, \\
\theta_{l} & =\pi-\frac{2 \pi l}{M},
\end{aligned}\right.
$$

for PSK constellations, respectively. $\Phi_{\Lambda, k \mid \cdot, \gamma}(s)$ is the Laplace transform of $f_{\Lambda, k \mid \cdot \gamma}(\lambda), 1 \leq k \leq 6$. Using the expressions for $f_{\Lambda, k \mid \cdot, \gamma}(\lambda)$ from Table I, the $\Phi_{\Lambda, k \mid \cdot, \gamma}(s)$ can be written as weighted sum of the following integrals:

$$
\begin{aligned}
I_{1 \mid \mu}(s) & =\int_{-\infty}^{\infty} \mathcal{N}_{\mu, 2 \mu}(x) \operatorname{erf}\left(\frac{x-\mu}{2 \sqrt{\mu}}\right) u(\mu-x) \exp (-s x) \mathrm{d} x, \\
I_{2 \mid \mu, \nu}(s) & =\int_{-\infty}^{\infty} \mathcal{N}_{\mu, 2 \mu}(x) \operatorname{erf}\left(\nu \frac{x-\mu}{\sqrt{\mu}}\right) \exp (-s x) \mathrm{d} x,
\end{aligned}
$$

where $\mu=d^{2} \gamma$ and $\nu=\tan (\theta / 2)$. Closed-form expressions for these integrals assuming $s \in \mathbb{R}^{+}$are derived in the appendix, and the resulting expressions for $\Phi_{\Lambda, k \mid \cdot \gamma}(s)$ are summarized in Table I.

2) Nakagami-m Fading Channel: We now consider the faded AWGN channel. Due to the linearity property of the Laplace transform, we have

$$
\Phi_{\Lambda \mid \bar{\gamma}, m}(s)=\int_{0}^{\infty} f_{\gamma \mid \bar{\gamma}, m}(\gamma) \Phi_{\Lambda \mid \gamma}(s) \mathrm{d} \gamma
$$

Substituting (22) and (23) for $\Phi_{\Lambda \mid \gamma}(s)$ in (26), we can write $\Phi_{\Lambda \mid \bar{\gamma}, m}(s)$ as linear superposition of

$$
\Phi_{\Lambda, k \mid, \bar{\gamma}, m}(s)=\int_{0}^{\infty} f_{\gamma \mid \bar{\gamma}, m}(\gamma) \Phi_{\Lambda, k \mid \cdot, \gamma}(s) \mathrm{d} \gamma
$$


for which expressions are given in Table I in terms of the integrals

$$
\begin{aligned}
I_{3 \mid \mu, \nu}(s) & =\int_{0}^{\infty} f_{\gamma \mid \bar{\gamma}, m}(\gamma) \exp \left(-\mu\left(s-s^{2}\right) \gamma\right) \operatorname{erf}(\nu s \sqrt{\gamma}) \mathrm{d} \gamma, \\
I_{4 \mid \mu, \nu}(s) & =\int_{0}^{\infty} f_{\gamma \mid \bar{\gamma}, m}(\gamma) \exp \left(-\mu\left(s-s^{2}\right) \gamma\right)(\operatorname{erf}(\nu s \sqrt{\gamma}))^{2} \mathrm{~d} \gamma .
\end{aligned}
$$

Here, $\mu=d^{2}$, and $\nu \in\{d / \sqrt{2}, d, d \sin (\theta / 2)\}$. In the appendix, we provide closed-form expressions for these integrals for $s \in \mathbb{R}^{+}$and general $m$ in terms of Appell's double Hypergeometric function and Gauss' Hypergeometric function [22] [23] together with simplified approximations in terms of elementary functions. Furthermore, for integer values of $m$ exact closed-form expressions are given using only elementary functions. For example, for the important case of Rayleigh fading $(m=1)$ the integrals in (28) and (29) are obtained as

$$
\begin{aligned}
I_{3 \mid \mu, \nu}(s) & =\frac{\nu s}{\left[1+\bar{\gamma} \mu\left(s-s^{2}\right)\right] \sqrt{(\nu s)^{2}+\mu\left(s-s^{2}\right)+\frac{1}{\bar{\gamma}}}}, \\
I_{4 \mid \mu, \nu}(s) & =\frac{4}{\pi\left[1+\bar{\gamma} \mu\left(s-s^{2}\right)\right]} \tan ^{-1}\left(\frac{\nu s}{\sqrt{(\nu s)^{2}+\mu\left(s-s^{2}\right)+\frac{1}{\bar{\gamma}}}}\right) .
\end{aligned}
$$

\section{Asymptotic AnAlysis for High SNRs}

In this section, we consider the case of asymptotically high SNR to further simplify the expressions for the PDF of LLRs and its Laplace transform. We then provide the saddlepoint approximation (8) and the direct derivation of the PEP without saddlepoint approximation for the asymptotic case. From this analysis we immediately obtain important performance indicators for BICM transmission over Nakagami- $m$ fading channels.

\section{A. PDF of LLRs and Its Laplace Transform}

The expressions for the PDF of LLRs and its Laplace transform for transmission over the nonfading AWGN channel shown in Table I can be simplified for high SNRs by replacing the error function with its asymptotic values, i.e., $\operatorname{erf}(x) \approx 1(-1)$ for $x \gg 0(x \ll 0)$, which corresponds to high SNRs $\gamma$. The Laplace transform expressions for transmission over fading channels are then obtained from averaging using (27). Table III summarizes the closed-form asymptotic results $f_{\Lambda, k \mid d, \gamma}^{\mathrm{a}}(\lambda), \Phi_{\Lambda, k \mid d, \gamma}^{\mathrm{a}}(s)$, 
and $\Phi_{\Lambda, k \mid d, \bar{\gamma}, m}^{\mathrm{a}}(s)$ for $1 \leq k \leq 6$. Using the expressions from Table III in (20)-(23), the following simplified expressions are obtained:

$$
\begin{aligned}
f_{\Lambda \mid c=1, \gamma}^{\mathrm{a}}(\lambda) & =\sum_{l=1}^{l_{\max }} N_{l} \mathcal{N}_{d_{l}^{2} \gamma, 2 d_{l}^{2} \gamma}(\lambda), \\
\Phi_{\Lambda \mid \gamma}^{\mathrm{a}}(s) & =\sum_{l=1}^{l_{\max }} N_{l} \exp \left(d_{l}^{2} \gamma\left(s^{2}-s\right)\right), \\
\Phi_{\Lambda \mid \bar{\gamma}, m}^{\mathrm{a}}(s) & =\sum_{l=1}^{l_{\max }} N_{l}\left(\frac{m}{m-d_{l}^{2} \bar{\gamma}\left(s^{2}-s\right)}\right)^{m},
\end{aligned}
$$

where

$$
\begin{aligned}
l_{\max } \triangleq \begin{cases}q_{\max }, & \text { for QAM, } \\
M / 2, & \text { for PSK, }\end{cases} \\
N_{l} \triangleq \begin{cases}\frac{2}{r M}\left(n_{1, l}+2 n_{2, l}+2 n_{3, l}+3 n_{4, l}+4 n_{5, l}\right), & \text { for QAM, } \\
\frac{2}{r M}\left(n_{1, l}+2 n_{6, l}\right), & \text { for PSK. }\end{cases}
\end{aligned}
$$

\section{B. Saddlepoint Approximation}

We now apply the saddlepoint approximation (8) and the asymptotic Laplace transform expressions from (31) and (32) to obtain asymptotic BER expressions. We note from the expressions for $\Phi_{\Lambda \mid \cdot(}^{\mathrm{a}}(s)$ in Table III that the saddlepoint $\hat{s}=1 / 2$ (cf. (9)).

1) Nonfading Channel: In the nonfading channel case $(m \rightarrow \infty, \gamma=\bar{\gamma})$, substituting the Laplace transform expression given in (31) into (8) and considering only the asymptotically dominant term, we arrive at

$$
\operatorname{PEP}\left(d_{\mathrm{H}} \mid \gamma\right)=\frac{N_{1}^{d_{\mathrm{H}}}}{d_{1} \sqrt{\pi d_{\mathrm{H}} \gamma}} \exp \left(-\frac{1}{4} d_{\mathrm{H}} d_{1}^{2} \gamma\right) .
$$

Finally, substituting (35) into (5) and only considering the PEP with minimum Hamming distance gives asymptotic BER for transmission over the nonfading channel.

2) Nakagami-m Fading Channel: The substitution of (32) into (8) results in

$$
\operatorname{PEP}\left(d_{\mathrm{H}} \mid \bar{\gamma}, m\right)=\frac{1}{2 \sqrt{\pi d_{\mathrm{H}} m}}\left[\sum_{l=1}^{l_{\max }}\left(\frac{N_{l}}{d_{l}^{2 m}}\right)\right]^{d_{\mathrm{H}}}\left(\frac{4 m}{\bar{\gamma}}\right)^{m d_{\mathrm{H}}} .
$$

Substituting (36) into (5) we can identify the diversity order as

$$
G_{d}=m d_{\mathrm{H}, \min }
$$


i.e., the product of the free distance of the convolutional code and the Nakagami- $m$ fading parameter. The horizontal offset of the log-error-rate curve, and thus the coding gain, depends on modulation, labeling, and fading parameter $m$. In particular, the coefficient

$$
d_{h \mid m}^{2} \triangleq\left[\sum_{l=1}^{l_{\max }}\left(\frac{N_{l}}{d_{l}^{2 m}}\right)\right]^{-1 / m}
$$

can be considered as a direct generalization of the harmonic mean $d_{h}^{2}$ obtained in [2, Eq. (63)] for Gray labeling and transmission over Rayleigh fading channels $(m=1)$ to arbitrary labeling rules and fading factors $m$.

\section{Direct Analysis}

The simplified expression (31) also allows direct evaluation of the PEP without saddlepoint approximation.

1) Nonfading Channel: For transmission over the nonfading AWGN channel we use (31) to define

$$
\Phi_{\Delta_{d_{\mathrm{H}}} \mid \gamma}(s) \triangleq\left[\Phi_{\Lambda \mid \gamma}^{\mathrm{a}}(s)\right]^{d_{\mathrm{H}}}=\left[\sum_{l=1}^{l_{\max }} N_{l} \exp \left(d_{l}^{2} \gamma\left(s^{2}-s\right)\right)\right]^{d_{\mathrm{H}}}
$$

Using the multinomial-series representation (cf. [22, p. 823]), this can be written as

$$
\Phi_{\Delta_{d_{\mathrm{H}}} \mid \gamma}(s)=\sum_{\substack{i_{1}, i_{2}, \ldots, i_{\max } \\ i_{1}+i_{2}+\ldots+i_{l_{\max }}=d_{\mathrm{H}}}}\left(\frac{d_{\mathrm{H}} !}{\prod_{l=1}^{l_{\max }} i_{l} !}\right)\left(\prod_{l=1}^{l_{\max }} N_{l}^{i_{l}}\right) \exp \left[\left(\sum_{l=1}^{l_{\max }} i_{l} d_{l}^{2}\right)\left(s^{2}-s\right) \gamma\right] .
$$

Since (40) is the Laplace transform of a linear superposition of Gaussian PDFs, the PEP is obtained in closed form as

$$
\operatorname{PEP}\left(d_{\mathrm{H}} \mid \gamma\right)=\sum_{\substack{i_{1}, i_{2}, \ldots, i_{l \max } \\ i_{1}+i_{2}+\ldots+i_{\max }=d_{\mathrm{H}}}}\left(\frac{d_{\mathrm{H}} !}{\prod_{l=1}^{l_{\max }} i_{l} !}\right)\left(\prod_{l=1}^{l_{\max }} N_{l}^{i_{l}}\right) Q\left[\sqrt{\frac{\gamma}{2}\left(\sum_{l=1}^{l_{\max }} i_{l} d_{l}^{2}\right)}\right] .
$$

Considering only the asymptotically dominant term gives the approximation

$$
\operatorname{PEP}\left(d_{\mathrm{H}} \mid \gamma\right)=N_{1}^{d_{\mathrm{H}}} Q\left(\sqrt{d_{\mathrm{H}} d_{1}^{2} \frac{\gamma}{2}}\right)
$$

Hence, the asymptotic PEP is expressed as the PEP for binary (i.e., BPSK) transmission with an equivalent SNR of $d_{\mathrm{H}} d_{1}^{2} \gamma$, scaled by a constant which is a function of the minimum Hamming 
distance of the code and the labeling rule. From the convergence of lower and upper bounds for the $Q$-function for large arguments [24], we obtain for $\gamma \rightarrow \infty$

$$
\operatorname{PEP}\left(d_{\mathrm{H}} \mid \gamma\right)=\frac{N_{1}^{d_{\mathrm{H}}}}{d_{1} \sqrt{\pi d_{\mathrm{H}} \gamma}} \exp \left(-\frac{1}{4} d_{\mathrm{H}} d_{1}^{2} \gamma\right)
$$

which coincides with the result (35) from the saddlepoint approximation.

2) Nakagami-m Fading Channel: Instead of directly using (32) we found it easier (i) first to determine the PEP conditioned on the vector $\gamma \triangleq\left[\gamma_{1}, \ldots, \gamma_{d_{\mathrm{H}}}\right]$ of instantaneous SNRs experienced by the $d_{\mathrm{H}}$ bits involved in an error event and (ii) then to average with respect to the PDF $f_{\gamma \mid \bar{\gamma}, m}(\gamma)$ of the SNR vector. Hence, starting again from (31) we write the Laplace transform conditioned on $\gamma$

$$
\Phi_{\Delta_{d_{\mathrm{H}}} \mid \boldsymbol{\gamma}}(s) \triangleq \prod_{i=1}^{d_{\mathrm{H}}} \Phi_{\Lambda \mid \gamma_{i}}^{\mathrm{a}}(s)=\sum_{\substack{\left\{l_{1}, \ldots, l_{d_{\mathrm{H}}}\right\} \\ \in\left\{1, \ldots, l_{\max }\right\}_{\mathrm{H}}}}\left(\prod_{j=1}^{d_{\mathrm{H}}} N_{l_{j}}\right) \exp \left(\sum_{j=1}^{d_{\mathrm{H}}} d_{l_{j}}^{2} \gamma_{j}\left(s^{2}-s\right)\right)
$$

from which we obtain the conditioned PEP as

$$
\operatorname{PEP}\left(d_{\mathrm{H}} \mid \gamma\right)=\sum_{\substack{\left\{l_{1}, \ldots, l_{d_{\mathrm{H}}}\right\} \\ \in\left\{1, \ldots, l_{\max }\right\}^{d_{\mathrm{H}}}}}\left(\prod_{j=1}^{d_{\mathrm{H}}} N_{l_{j}}\right) Q\left(\sqrt{\frac{1}{2} \sum_{j=1}^{d_{\mathrm{H}}} d_{l_{j}}^{2} \gamma_{j}}\right) .
$$

Applying the alternative representation of the Q-function (cf. [14, p. 85]) and averaging with respect to the SNR vector leads to

$$
\operatorname{PEP}\left(d_{\mathrm{H}} \mid \bar{\gamma}, m\right)=\frac{1}{\pi} \sum_{\substack{\left\{l_{1}, \ldots, l_{d_{\mathrm{H}}}\right\} \\ \in\left\{1, \ldots, l_{\max }\right\}_{\mathrm{H}}}}\left(\prod_{j=1}^{d_{\mathrm{H}}} N_{l_{j}}\right) \int_{0}^{\pi / 2} \prod_{j=1}^{d_{\mathrm{H}}}\left(1+\frac{d_{l_{j}}^{2} \bar{\gamma}}{4 m \sin ^{2}(\phi)}\right)^{-m} \mathrm{~d} \phi
$$

Making the high SNR assumption $1 \ll \frac{d_{l_{j}}^{2} \bar{\gamma}}{4 m \sin ^{2}(\phi)}$, we obtain

$$
\operatorname{PEP}\left(d_{\mathrm{H}} \mid \bar{\gamma}, m\right)=\left(\sum_{l=1}^{l_{\max }} \frac{N_{l}}{d_{l}^{2 m}}\right)^{d_{\mathrm{H}}}\left(\frac{4 m}{\bar{\gamma}}\right)^{m d_{\mathrm{H}}} \frac{1}{\pi} \int_{0}^{\pi / 2}(\sin \phi)^{2 m d_{\mathrm{H}}} \mathrm{d} \phi,
$$

which finally can be solved to [25, Eq. 3.621]

$$
\operatorname{PEP}\left(d_{\mathrm{H}} \mid \bar{\gamma}, m\right)=\frac{\Gamma\left(m d_{\mathrm{H}}+1 / 2\right)}{2 \sqrt{\pi} \Gamma\left(m d_{\mathrm{H}}+1\right)}\left(\sum_{l=1}^{l_{\max }} \frac{N_{l}}{d_{l}^{2 m}}\right)^{d_{\mathrm{H}}}\left(\frac{4 m}{\bar{\gamma}}\right)^{m d_{\mathrm{H}}} .
$$

We note that the PEP in (48) has the same form as the PEP (36) obtained with the saddlepoint approximation. In particular, the diversity order $G_{d}(37)$ and the generalized harmonic mean $d_{h \mid m}^{2}(38)$ 
are confirmed as important parameters for code and channel diversity and coding gain. Furthermore, recalling the asymptotic series

$$
\frac{\Gamma(x+1 / 2)}{\Gamma(x+1)}=x^{-1 / 2}\left[1+O\left(x^{-1}\right)\right], \quad x \rightarrow \infty,
$$

we note that (48) and (36) become identical for $m d_{\mathrm{H}} \gg 1$. The discrepancy for small values of $m d_{\mathrm{H}}$ indicates the insufficiency of the second-order approximation of the cumulant transform $\log \left(\Phi_{\Lambda \mid \bar{\gamma}, m}(-s)\right)$ used in (8) for extreme cases of fading (i.e., $m \ll 1$ ).

\section{Numerical Results AND Discussions}

In this section, we present a number of exemplary numerical results to illustrate the accuracy of cutoff rate and BER approximations based on the new closed-form expressions. For the BER results we assume BICM with the popular, quasi-standard 64-state rate-1/2 convolutional code with generator polynomials $(171,133)_{8}$.

1) Cutoff Rate Results: First, we consider the cutoff rate (12) using the closed-form approximations for the Laplace transform derived in Section III-B. We found that $s=1 / 2$ yields practically the same results as when using the exact saddlepoint $\hat{s}$ (cf. Eq. (9)), which is consistent with the results reported in [11], [12] and the fact that $\hat{s} \rightarrow 1 / 2$ with increasing SNR (cf. Section IV-B). We therefore adopted $s=1 / 2$ in all cases.

Fig. 4 shows $R_{0}$-curves for QAM and PSK constellations with different labeling rules and channel types. In addition to GL, ML and SPL (cf. Section II-A) are also included. The markers represent $R_{0}$-values obtained with Monte Carlo simulation, and the lines represent results from the evaluation of the closed form expression. We observe that there is an excellent agreement between analytical and simulation results for a wide range of SNR, and in particular for all $R_{0}$ values of practical interest. The discrepancies between analytical and simulated cutoff-rate curves for non-GL and low SNRs in Fig. 4 are expected, since the underlying approximation of the PDF of LLRs (cf. Section III) is not accurate in this SNR range. In fact, for non-GL the $R_{0}$ values can drop below zero, as the proposed approximation excludes neighboring signal points with the same binary label as the transmitted bit and thus overestimates the probability of error in very low SNRs. 
2) BER Results: Next, we compare simulated and analytical BER results as function of the bitwise SNR $\bar{\gamma}_{\mathrm{b}} \triangleq \bar{\gamma} /\left(r R_{c}\right)$. In case of the BER union bound (5), only the 15 first terms of the distance spectrum of the convolutional code were taken into account, and thus, strictly speaking, the BER union bound is a BER approximation.

Fig. 5 shows the analytical (lines) and simulated (markers) BER results for different constellations and labeling for transmission over the nonfading AWGN channel. Solid lines represent the BER union bound, while dashed lines represent the asymptotic approximation (42) for $d_{\mathrm{H}}=d_{\mathrm{H}, \min }$, i.e., only the asymptotically dominating error event is considered. We observe that the BER union bound is fairly tight for all modulation schemes and BERs below about $10^{-4}$. Likewise, the proposed simple expression (42) accurately predicts the asymptotic error-rate performance at high SNR. Similar results are obtained with the expression (35) derived from the saddlepoint approximation, which is apparent from the equivalence of (42) and (35) for high SNR (cf. Section IV-C).

We now compare analytical and simulated BER results for BICM transmission over fading channels with different constellations and labeling rules. To this end, Fig. 6 shows BER curves obtained from the BER union bound and the exact closed-form solutions for integrals (28), (29) (solid lines) and their approximations (62), (67) (dashed lines) derived in the appendix. Again, we observe an excellent match between results from analysis and simulations, which confirms the validity of the approximations made for the derivation of the closed-form BER expressions. Since this is also true for the expressions using the exponential approximations of the error function, i.e., (62) and (67), we have provided tight BER approximations in terms of elementary functions.

Finally, in Fig. 7 the asymptotic BER results from (48) and $d_{\mathrm{H}}=d_{\mathrm{H}, \min }$ (solid lines) are plotted together with the BER union bound (markers) for the same transmission scenarios as in Fig. 6. It can be seen that the asymptotic results correctly predict coding and fading gain of the BICM scheme. Similar results are obtained when evaluating (36), since the term on the left-hand side of (49) is well approximated by $\left(m d_{\mathrm{H}}\right)^{-1 / 2}$ for $m d_{\mathrm{H}}=m d_{\mathrm{H}, \min }=10 m \geq 5$ for $m \in\{0.5,1\}$ in Fig. 7. Hence, we conclude that the simple expressions (36) and (48) are very valuable to quickly determine the asymptotic performance of BICM transmission over fading channels. 


\section{CONCLUSION}

In this paper we have presented a generalized method for analyzing the performance of BICM transmission. Its key element is a new approximation of the PDF of the bitwise reliability metrics, which is a valuable contribution in its own right. This approximation has led us to closed-form expressions for the Laplace transform of the PDF, in terms of which BER and cutoff rate of BICM can be expressed. Notably, our results are applicable to BICM with arbitrary QAM and PSK constellations and labeling rules, and transmission over Nakagami- $m$ fading channels for arbitrary $m$. Furthermore, we have developed an asymptotic analysis which provides valuable insights into the performance of BICM over fading channels, namely expressions for diversity order and asymptotic coding gain. Selected numerical results have confirmed the accuracy of the proposed analytical results for SNR regions of interest for convolutional coded BICM. We believe that the developed expressions for nonGray labeling are not only of theoretical interest, but bear significance for BICM transmission with iterative decoding, for which the BER approximation predicts performance after the first decoding iteration and the generalized harmonic mean enables optimization of the labeling rule [15].

\section{APPENDIX}

In this appendix, we present the solutions for the four integrals $I_{k \mid \cdot}(s)$ that appear in Section III-B.

\section{A. Closed-form Expression for $I_{1 \mid \mu}(s)$ in (24) for $s \in \mathbb{R}^{+}$}

Using the integral form of the error function (19), $I_{1 \mid \mu}(s)$ from (24) is written as

$$
I_{1 \mid \mu}(s)=\frac{1}{\pi \sqrt{\mu}} \int_{-\infty}^{\mu} \int_{0}^{\frac{x-\mu}{2 \sqrt{\mu}}} \exp \left(\frac{(x-\mu)^{2}}{4 \mu}\right) \exp \left(-x_{2}^{2}\right) \exp (-s x) d x_{2} \mathrm{~d} x .
$$

Applying the change of variables $x_{1} \triangleq \frac{x+\mu(2 s-1)}{2 \sqrt{\mu}}$ leads to

$$
I_{1 \mid \mu}(s)=-\frac{2}{\pi} \exp \left(\mu\left(s^{2}-s\right)\right) \int_{-\infty}^{\sqrt{\mu} s} \int_{0}^{\sqrt{\mu} s-x_{1}} \exp \left(-\left(x_{1}^{2}+x_{2}^{2}\right)\right) d x_{2} d x_{1} .
$$

The support of this integral is illustrated in Fig. 8 (shaded area in the left sub-figure), from which we can express it as

$$
I_{1 \mid \mu}(s)=-\frac{2}{\pi} \exp \left(\mu\left(s^{2}-s\right)\right)\left(S_{1}+S_{2}+\frac{S_{3}}{2}\right)
$$


where $S_{1}, S_{2}$, and $S_{3}$ denote the corresponding areas indicated in the Fig. 8 (left sub-figure). Using the rotational invariance of the integrand in (51), the areas are easily determined and from (52) the integral is finally obtained as

$$
I_{1 \mid \mu}(s)=-\frac{1}{4} \exp \left(\mu\left(s^{2}-s\right)\right)\left(1+\operatorname{erf}\left(\sqrt{\frac{\mu}{2}} s\right)\right)^{2} .
$$

B. Closed-form Expression for $I_{2 \mid \mu, \nu}(s)$ in (25) for $s \in \mathbb{R}^{+}$

Starting from (25) and performing the same transformations as above, we obtain

$$
I_{2 \mid \mu, \nu}(s)=-\frac{2}{\pi} \exp \left(\mu\left(s^{2}-s\right)\right) \int_{-\infty}^{\infty} \int_{0}^{2 \nu\left(\sqrt{\mu} s-x_{1}\right)} \exp \left(-\left(x_{1}^{2}+x_{2}^{2}\right)\right) d x_{2} d x_{1} .
$$

The support of this integral is illustrated in Fig. 8 (shaded area in the right sub-figure). Exploiting again the fact that integrand in (51) is rotational invariant, we can write

$$
I_{2 \mid \mu, \nu}(s)=-\frac{2}{\pi} \exp \left(\mu\left(s^{2}-s\right)\right)\left(\frac{S_{4}}{2}\right),
$$

where $S_{4}$ is illustrated in Fig. 8 (right sub-figure). Finally, we arrive at the closed-form expression

$$
I_{2 \mid \mu, \nu}(s)=-\exp \left(\mu\left(s^{2}-s\right)\right) \operatorname{erf}\left(\frac{2 \nu}{\sqrt{1+(2 \nu)^{2}}} \sqrt{\mu} s\right) \text {. }
$$

C. Computation of $I_{3 \mid \mu, \nu}(s)$ in (28) for $s \in \mathbb{R}^{+}$

1) Exact Solution: Applying the alternative representation of the Q-function (cf. [14, p. 85]), the integral (28) can be written as

$$
I_{3 \mid \mu, \nu}(s)=\left(\frac{m}{m+\bar{\gamma} \mu\left(s-s^{2}\right)}\right)^{m}\left[1-2 P_{1}\right]
$$

where

$$
P_{1} \triangleq \frac{1}{\pi} \int_{0}^{\frac{\pi}{2}}\left(1+\frac{c}{\sin ^{2}(\varphi)}\right)^{-m} d \varphi, \quad c \triangleq \frac{(\nu s)^{2}}{\frac{m}{\bar{\gamma}}+\mu\left(s-s^{2}\right)} .
$$

The integral $P_{1}$ has been solved in [14, p. 127] as

$$
\mathcal{P}_{1}=\frac{1}{2 \sqrt{\pi}} \frac{\sqrt{c}}{(1+c)^{(m+0.5)}} \frac{\Gamma\left(m+\frac{1}{2}\right)}{\Gamma(m+1)}{ }_{2} F_{1}\left(1, m+\frac{1}{2} ; m+1 ; \frac{1}{1+c}\right) \text {. }
$$


for general values of $m$ in terms of the Gauss Hypergeometric function ${ }_{2} F_{1}(\cdot, \cdot ; \cdot ; \cdot)$, which simplifies to

$$
P_{1}=\frac{1}{2}\left[1-p(c) \sum_{k=0}^{m-1}\left(\begin{array}{c}
2 k \\
k
\end{array}\right)\left(\frac{1-[p(c)]^{2}}{4}\right)^{k}\right], \quad p(c) \triangleq \sqrt{\frac{c}{1+c}},
$$

for positive integer $m$. Substituting (59) or (60) into (57) gives the desired closed form.

2) Approximation: For non-integer $m$ an approximation of $I_{3 \mid \mu, \nu}(s)$ in terms of elementary functions may be desirable. This is possible through the use of the exponential approximations of erf $(x)$. For example, using the tight approximation [26]

$$
\operatorname{erf}(x) \approx 1-\frac{1}{6} \exp \left(-x^{2}\right)-\frac{1}{2} \exp \left(\frac{-4 x^{2}}{3}\right)
$$

the integral (28) can be approximated as

$$
I_{3 \mid \mu, \nu}(s) \approx \sum_{i=1}^{3} a_{i}\left(\frac{m}{m+\left[\mu\left(s-s^{2}\right)+b_{i}(\nu s)^{2}\right] \bar{\gamma}}\right)^{m},
$$

where $\left[a_{1}, a_{2}, a_{3}\right]=\left[1,-\frac{1}{6},-\frac{1}{2}\right]$ and $\left[b_{1}, b_{2}, b_{3}\right]=\left[0,1, \frac{4}{3}\right]$.

\section{Computation of $I_{4 \mid \mu, \nu}(s)$ in (29) for $s \in \mathbb{R}^{+}$}

1) Exact Solution: Using again the alternative representation of the Q-function (cf. [14, p. 85]), we can rewrite the integral (29) as

$$
I_{4 \mid \mu, \nu}(s)=\left(\frac{m}{m+\bar{\gamma} \mu\left(s-s^{2}\right)}\right)^{m}\left[1-4 P_{1}+4 P_{2}\right],
$$

where $P_{1}$ and $c$ are defined in (58), and

$$
P_{2} \triangleq \frac{1}{\pi} \int_{0}^{\frac{\pi}{4}}\left(1+\frac{c}{\sin ^{2}(\varphi)}\right)^{-m} d \varphi
$$

The integral $P_{2}$ has been computed in [27, p.538] for general $m$ in terms of Appell's double Hypergeometric function $F_{1}(\cdot ; \cdot, \cdot ; \cdot ; \cdot, \cdot)$ :

$$
P_{2}=\frac{1}{2 \pi(2 m+1)}\left(\frac{1}{1+2 c}\right) F_{1}\left(1 ; m, 1 ; m+\frac{3}{2} ; \frac{1+c}{1+2 c}, \frac{1}{2}\right),
$$


In case of positive integer $m$, a closed-form expression in terms of elementary functions can be obtained [14, p. 130]:

$$
\begin{aligned}
P_{2}= & \frac{1}{4}-\frac{1}{\pi} p(c)\left\{\left(\frac{\pi}{2}-\tan ^{-1}(p(c))\right) \sum_{k=0}^{m-1}\left[\left(\begin{array}{c}
2 k \\
k
\end{array}\right) \frac{1}{(4(1+c))^{k}}\right]\right. \\
& \left.-\sin \left(\tan ^{-1}(p(c))\right) \sum_{k=1}^{m-1} \sum_{i=1}^{k}\left[\frac{T_{i k}}{(1+c)^{k}}\left[\cos \left(\tan ^{-1}(p(c))\right)\right]^{2(k-i)+1}\right]\right\},
\end{aligned}
$$

where $T_{i k} \triangleq \frac{\left(\begin{array}{c}2 k \\ k\end{array}\right)}{\left(\begin{array}{c}2(k-i) \\ k-i\end{array}\right) 4^{i}[2(k-i)+1]}$.

2) Approximation: Exponential approximations of erf $(x)$ allow us to express $I_{4 \mid \mu, \nu}(s)$ in terms of elementary functions also for non-integer $m$. For example, applying again approximation (61), we obtain

$$
I_{4 \mid \mu, \nu}(s) \approx \sum_{i=1}^{6} a_{i}\left(\frac{m}{m+\left[\mu\left(s-s^{2}\right)+b_{i}(\nu s)^{2}\right] \bar{\gamma}}\right)^{m},
$$

where $\left[a_{1}, a_{2}, a_{3}, a_{4}, a_{5}, a_{6}\right]=\left[1,-\frac{1}{3},-1, \frac{1}{36}, \frac{1}{6}, \frac{1}{4}\right]$ and $\left[b_{1}, b_{2}, b_{3}, b_{4}, b_{5}, b_{6}\right]=\left[0,1, \frac{4}{3}, 2, \frac{7}{3}, \frac{8}{3}\right]$.

\section{REFERENCES}

[1] E. Zehavi, "8-PSK trellis codes for a Rayleigh channel," IEEE Trans. Commun., vol. 40, no. 5, pp. 873-884, May 1992.

[2] G. Caire, G. Taricco, and E. Biglieri, "Bit-interleaved coded modulation," IEEE Trans. Inform. Theory, vol. 44, no. 3, pp. 927-946, May 1998.

[3] G. Ungerboeck, "Channel coding with multilevel/phase signals," IEEE Trans. Inform. Theory, vol. 28, pp. 55-67, Jan. 1982.

[4] A. Guillén i Fàbregas, A. Martinez, and G. Caire, "Bit-interleaved coded modulation," Foundations and Trends in Communications and Information Theory, vol. 5, no. 1-2, pp. 1-153, 2008.

[5] V. Sethuraman and B. Hajek, "Comments on "Bit-interleaved coded modulation"," IEEE Trans. Inform. Theory, vol. 52, no. 4, pp. 1795-1797, Apr. 2006.

[6] P.-C. Yeh, S. Zummo, and W. Stark, "Error probability of bit-interleaved coded modulation in wireless environments," IEEE Trans. Veh. Technol., vol. 55, no. 2, pp. 722-728, March 2006.

[7] A. Martinez, A. Guillén i Fàbregas, and G. Caire, "Error probability analysis of bit-interleaved coded modulation," IEEE Trans. Inform. Theory, vol. 52, no. 1, pp. 262-271, Jan. 2006.

[8] C. Goutis and G. Casella, "Explaining the saddlepoint approximation,” The American Statistician, vol. 53, no. 3, pp. 216-224, Aug. 1999.

[9] L. Szczecinski, R. Bettancourt, and R. Feick, "Probability density function of reliability metrics in BICM with arbitrary modulation: Closed-form through algorithmic approach," IEEE Trans. Commun., vol. 56, no. 5, pp. 736-742, May 2008.

[10] A. Alvarado, L. Szczecinski, R. Feick, and L. Ahumada, "Distribution of L-values in Gray-mapped $M^{2}$-QAM: Closed-form approximations and applications," IEEE Trans. Commun., vol. 57, no. 7, pp. 2071-2079, July 2009. 
[11] L. Szczecinski, A. Alvarado, and R. Feick, "Distribution of max-log metrics for QAM-based BICM in fading channels," IEEE Trans. Commun., vol. 57, no. 9, Sept. 2009.

[12] — _ "Closed-form approximation of coded BER in QAM-based BICM faded transmission," IEEE Sarnoff Symposium, pp. 1-5, Apr. 2008.

[13] A. Martinez and A. Guillén i Fàbregas, "Large-SNR error probability analysis of BICM with uniform interleaving in fading channels," IEEE Trans. Wireless Commun., vol. 8, no. 1, pp. 38-44, Jan. 2009.

[14] M. K. Simon and M. S. Alouini, Digital Communication Over Fading Channels. New York: Wiley-Interscience, 2000.

[15] A. Chindapol and J. Ritcey, "Design, analysis, and performance evaluation for BICM-ID with square QAM constellations in Rayleigh fading channels," ”IEEE J. Select. Areas Commun.”, vol. 19, no. 5, pp. 944-957, May 2001.

[16] X. Li, A. Chindapol, and J. Ritcey, "Bit-interleaved coded modulation with iterative decoding and 8PSK signaling," IEEE Trans. Commun., vol. 50, no. 8, pp. 1250-1257, Aug 2002.

[17] E. Agrell, J. Lassing, E. Ström, and T. Ottosson, "On the optimality of the binary reflected Gray code," IEEE Trans. Inform. Theory, vol. 50, no. 12, pp. 3170-3182, Dec. 2004.

[18] B. Classon, K. Blankenship, and V. Desai, "Channel coding for 4G systems with adaptive modulation and coding," IEEE Trans. Wireless Commun., vol. 9, no. 2, pp. 8-13, April 2002.

[19] A. J. Viterbi and J. K. Omura, Principles of Digital Communication and Coding. New York: McGraw-Hill, Inc., 1979.

[20] E. Biglieri, G. Caire, G. Taricco, and J. Ventura-Traveset, "Computing error probabilities over fading channels: A unified approach," Eur. Trans. Telecommun., vol. 9, no. 1, pp. 15-25, Jan./Feb. 1998.

[21] C. Schlegel and D. Costello, Jr., "Bandwidth efficient coding for fading channels: Code construction and performance analysis," IEEE J. Select. Areas Commun., vol. 7, no. 9, pp. 1356-1368, Dec. 1989.

[22] M. Abramowitz and I. Stegun, Handbook of Mathematical Functions. New York: Dover Publications, 1972.

[23] R. Askey, "Multiple hypergeometric functions and applications (harold exton)," SIAM Review, vol. 20, no. 4, pp. 874-875, 1978. [Online]. Available: http://link.aip.org/link/?SIR/20/874/1

[24] T. Philips and A. Sahraoui, "A sequence of upper and lower bounds for the $Q$ function," IEEE Trans. Inform. Theory, vol. 30, no. 6, pp. 877-878, Nov. 1984.

[25] I. Gradshteyn and I. Ryzhik, Table of Integrals, Series, and Products, 7th ed. Academic Press, 2007.

[26] M. Chiani, D. Dardari, and M. Simon, "New exponential bounds and approximations for the computation of error probability in fading channels," IEEE Trans. Wireless Commun., vol. 2, no. 4, pp. 840-845, July 2003.

[27] H. Shin and J. H. Lee, "On the error probability of binary and M-ary signals in Nakagami-m fading channels," IEEE Trans. Commun., vol. 52, no. 4, pp. 536-539, April 2004. 
TABLE I

PROBABILITY DENSITY FUNCTION OF LLRS $f_{\Lambda, k \mid \cdot, \gamma}(\lambda)$ FOR TRANSMISSION OVER NONFADING AWGN CHANNEL, ITS LAPLACE TRANSFORM $\Phi_{\Lambda, k \mid \cdot, \gamma}(s)$, AND THE LAPLACE TRANSFORM OF THE PDF OF LLRS WHEN TRANSMITTING OVER NAKAGAMI- $m$ FADING CHANNELS $\Phi_{\Lambda, k \mid \cdot, \bar{\gamma}, m}(s)$ FOR THE SIX DIFFERENT SETS OF COMPETITIVE SIGNAL POINTS $\mathcal{A}_{j, x}$ SHOWN IN FIG. 2.

\begin{tabular}{|c|c|c|}
\hline \multirow{3}{*}{ Case 1} & $f_{\Lambda, 1 \mid d, \gamma}(\lambda)$ & $\mathcal{N}_{d^{2} \gamma, 2 d^{2} \gamma}(\lambda)$ \\
\hline & $\Phi_{\Lambda, 1 \mid d, \gamma}(s)$ & $\exp \left(d^{2} \gamma\left(s^{2}-s\right)\right)$ \\
\hline & $\Phi_{\Lambda, 1 \mid d, \bar{\gamma}, m}(s)$ & $\left(\frac{m}{m-d^{2} \bar{\gamma}\left(s^{2}-s\right)}\right)^{m}$ \\
\hline \multirow{3}{*}{ Case 2} & $f_{\Lambda, 2 \mid d, \gamma}(\lambda)$ & $\mathcal{N}_{d^{2} \gamma, 2 d^{2} \gamma}(\lambda)\left(1-\operatorname{erf}\left(\frac{\lambda-d^{2} \gamma}{2 d \sqrt{\gamma}}\right)\right)$ \\
\hline & $\Phi_{\Lambda, 2 \mid d, \gamma}(s)$ & $\exp \left(d^{2} \gamma\left(s^{2}-s\right)\right)\left(1+\operatorname{erf}\left(d \sqrt{\frac{\gamma}{2}} s\right)\right)$ \\
\hline & $\Phi_{\Lambda, 2 \mid d, \bar{\gamma}, m}(s)$ & $\left(\frac{m}{m-d^{2} \bar{\gamma}\left(s^{2}-s\right)}\right)^{m}+I_{3 \mid d^{2}, d / \sqrt{2}}(s)$ \\
\hline \multirow{3}{*}{ Case 3} & $f_{\Lambda, 3 \mid d, \gamma}(\lambda)$ & $2 \mathcal{N}_{d^{2} \gamma, 2 d^{2} \gamma}(\lambda) u\left(d^{2} \gamma-\lambda\right)$ \\
\hline & $\Phi_{\Lambda, 3 \mid d, \gamma}(s)$ & $\exp \left(d^{2} \gamma\left(s^{2}-s\right)\right)(1+\operatorname{erf}(d \sqrt{\gamma} s))$ \\
\hline & $\Phi_{\Lambda, 3 \mid d, \bar{\gamma}, m}(s)$ & $\left(\frac{m}{m-d^{2} \bar{\gamma}\left(s^{2}-s\right)}\right)^{m}+I_{3 \mid d^{2}, d}(s)$ \\
\hline \multirow{3}{*}{ Case 4} & $f_{\Lambda, 4 \mid d, \gamma}(\lambda)$ & $\mathcal{N}_{d^{2} \gamma, 2 d^{2} \gamma}(\lambda)\left(1-2 \operatorname{erf}\left(\frac{\lambda-d^{2} \gamma}{2 d \sqrt{\gamma}}\right)\right) u\left(d^{2} \gamma-\lambda\right)$ \\
\hline & $\Phi_{\Lambda, 4 \mid d, \gamma}(s)$ & $\frac{1}{2} \exp \left(d^{2} \gamma\left(s^{2}-s\right)\right)\left(1+\operatorname{erf}(d \sqrt{\gamma} s)+\left(1+\operatorname{erf}\left(d \sqrt{\frac{\gamma}{2}} s\right)\right)^{2}\right)$ \\
\hline & $\Phi_{\Lambda, 4 \mid d, \bar{\gamma}, m}(s)$ & $\left(\frac{m}{m-d^{2} \bar{\gamma}\left(s^{2}-s\right)}\right)^{m}+\frac{1}{2} I_{3 \mid d^{2}, d}(s)+I_{3 \mid d^{2}, d / \sqrt{2}}(s)+\frac{1}{2} I_{4 \mid d^{2}, d / \sqrt{2}}(s)$ \\
\hline \multirow{3}{*}{ Case 5} & $f_{\Lambda, 5 \mid d, \gamma}(\lambda)$ & $-4 \mathcal{N}_{d^{2} \gamma, 2 d^{2} \gamma}(\lambda) \operatorname{erf}\left(\frac{\lambda-d^{2} \gamma}{2 d \sqrt{\gamma}}\right) u\left(d^{2} \gamma-\lambda\right)$ \\
\hline & $\Phi_{\Lambda, 5 \mid d, \gamma}(s)$ & $\exp \left(d^{2} \gamma\left(s^{2}-s\right)\right)\left(1+\operatorname{erf}\left(d \sqrt{\frac{\gamma}{2}} s\right)\right)^{2}$ \\
\hline & $\Phi_{\Lambda, 5 \mid d, \bar{\gamma}, m}(s)$ & $\left(\frac{m}{m-d^{2} \bar{\gamma}\left(s^{2}-s\right)}\right)^{m}+2 I_{3 \mid d^{2}, d / \sqrt{2}}(s)+I_{4 \mid d^{2}, d / \sqrt{2}}(s)$ \\
\hline \multirow{3}{*}{ Case 6} & $f_{\Lambda, 6 \mid d, \theta, \gamma}(\lambda)$ & $\mathcal{N}_{d^{2} \gamma, 2 d^{2} \gamma}(\lambda)\left(1-\operatorname{erf}\left(\tan \left(\frac{\theta}{2}\right) \frac{\lambda-d^{2} \gamma}{2 d \sqrt{\gamma}}\right)\right)$ \\
\hline & $\Phi_{\Lambda, 6 \mid d, \theta, \gamma}(s)$ & $\exp \left(d^{2} \gamma\left(s^{2}-s\right)\right)\left(1+\operatorname{erf}\left(\sin \left(\frac{\theta}{2}\right) d \sqrt{\gamma} s\right)\right)$ \\
\hline & $\Phi_{\Lambda, 6 \mid d, \theta, \bar{\gamma}, m}(s)$ & $\left(\frac{m}{m-d^{2} \bar{\gamma}\left(s^{2}-s\right)}\right)^{m}+I_{3 \mid d^{2}, \sin (\theta / 2) d}(s)$ \\
\hline
\end{tabular}


TABLE II

NUMBERS $n_{k, l}, 1 \leq l \leq\left\{q_{\max }, M / 2\right\}$, OF NEAREST COMPETITIVE SIGNAL SETS OF TYPE $k, 1 \leq k \leq 6$ (CASES SHOWN IN FIG. 2) FOR DIFFERENT CONSTELLATIONS AND LABELINGS USED FOR NUMERICAL RESULTS IN SECTIONS III AND V. ONLY NON-ZERO COEFFICIENTS $n_{k, l}$ ARE SHOWN.

\begin{tabular}{|c|l|l|}
\hline \multirow{2}{*}{ 4QAM } & GL & $n_{1,1}=4$ \\
\cline { 2 - 3 } & SPL & $n_{1,1}=2, n_{2,1}=2$ \\
\hline \hline \multirow{3}{*}{ 16QAM } & GL & $n_{1,1}=24, n_{1,2}=8$ \\
\cline { 2 - 3 } & SPL & $n_{1,1}=8, n_{1,2}=4, n_{2,1}=10, n_{3,1}=4, n_{4,1}=4, n_{5,1}=2$ \\
\cline { 2 - 4 } & MSPL & $n_{1,1}=16, n_{2,1}=4, n_{2,2}=2, n_{3,1}=4, n_{4,1}=4, n_{5,1}=2$ \\
\cline { 2 - 4 } & ML & $n_{1,1}=24, n_{3,1}=8$ \\
\hline \hline \multirow{2}{*}{ 64QAM } & GL & $n_{1,1}=112, n_{1,2}=48, n_{1,3}=16, n_{1,4}=16$ \\
\hline \hline \multirow{2}{*}{ 8PSK } & SPL & $n_{1,1}=6, n_{1,2}=2, n_{2,1}=4$ \\
\cline { 2 - 4 } & GL & $n_{1,1}=8, n_{1,2}=4$ \\
\cline { 2 - 4 } & SSPL & $n_{1,1}=6, n_{2,1}=6$ \\
\hline
\end{tabular}


TABLE III

ASymptotic VALUes of Probability Density FUnCtion of LLRS $f_{\Lambda, k \mid d, \gamma}^{\mathrm{a}}(\lambda)$ FOR TRANSMission OVER NONFADING AWGN CHANNEL, ITS LAPLACE TRANSFORM $\Phi_{\Lambda, k \mid d, \gamma}^{\mathrm{a}}(s)$, AND THE LAPLACE TRANSFORM OF THE PDF OF LLRS WHEN TRANSMITTING OVER NAKAGAMI- $m$ FADING CHANNELS $\Phi_{\Lambda, k \mid d, \bar{\gamma}, m}^{\mathrm{a}}(s)$ FOR THE SIX DIFFERENT SETS OF COMPETITIVE SIGNAL POINTS $\mathcal{A}_{j, x}$ SHOWN IN FIG. 2.

\begin{tabular}{|c|c|c|}
\hline \multirow{3}{*}{ Case 1} & $f_{\Lambda, 1 \mid d, \gamma}^{\mathrm{a}}(\lambda)$ & $\mathcal{N}_{d^{2} \gamma, 2 d^{2} \gamma}(\lambda)$ \\
\hline & $\Phi_{\Lambda, 1 \mid d, \gamma}^{\mathrm{a}}(s)$ & $\exp \left(d^{2} \gamma\left(s^{2}-s\right)\right)$ \\
\hline & $\Phi_{\Lambda, 1 \mid d, \bar{\gamma}, m}^{\mathrm{a}}(s)$ & $\left(\frac{m}{m-d^{2} \bar{\gamma}\left(s^{2}-s\right)}\right)^{m}$ \\
\hline \multirow{3}{*}{ Case 2} & $f_{\Lambda, 2 \mid d, \gamma}^{\mathrm{a}}(\lambda)$ & $2 \mathcal{N}_{d^{2} \gamma, 2 d^{2} \gamma}(\lambda)$ \\
\hline & $\Phi_{\Lambda, 2 \mid d, \gamma}^{\mathrm{a}}(s)$ & $2 \exp \left(d^{2} \gamma\left(s^{2}-s\right)\right)$ \\
\hline & $\Phi_{\Lambda, 2 \mid d, \bar{\gamma}, m}^{\mathrm{a}}(s)$ & $2\left(\frac{m}{m-d^{2} \bar{\gamma}\left(s^{2}-s\right)}\right)^{m}$ \\
\hline \multirow{3}{*}{ Case 3} & $f_{\Lambda, 3 \mid d, \gamma}^{\mathrm{a}}(\lambda)$ & $2 \mathcal{N}_{d^{2} \gamma, 2 d^{2} \gamma}(\lambda)$ \\
\hline & $\Phi_{\Lambda, 3 \mid d, \gamma}^{\mathrm{a}}(s)$ & $2 \exp \left(d^{2} \gamma\left(s^{2}-s\right)\right)$ \\
\hline & $\Phi_{\Lambda, 3 \mid d, \bar{\gamma}, m}^{\mathrm{a}}(s)$ & $2\left(\frac{m}{m-d^{2} \bar{\gamma}\left(s^{2}-s\right)}\right)^{m}$ \\
\hline \multirow{3}{*}{ Case 4} & $f_{\Lambda, 4 \mid d, \gamma}^{\mathrm{a}}(\lambda)$ & $3 \mathcal{N}_{d^{2} \gamma, 2 d^{2} \gamma}(\lambda)$ \\
\hline & $\Phi_{\Lambda, 4 \mid d, \gamma}^{\mathrm{a}}(s)$ & $3 \exp \left(d^{2} \gamma\left(s^{2}-s\right)\right)$ \\
\hline & $\Phi_{\Lambda, 4 \mid d, \bar{\gamma}, m}^{\mathrm{a}}(s)$ & $3\left(\frac{m}{m-d^{2} \bar{\gamma}\left(s^{2}-s\right)}\right)$ \\
\hline \multirow{3}{*}{ Case 5} & $f_{\Lambda, 5 \mid d, \gamma}^{\mathrm{a}}(\lambda ; d, \gamma)$ & $4 \mathcal{N}_{d^{2} \gamma, 2 d^{2} \gamma}(\lambda)$ \\
\hline & $\Phi_{\Lambda, 5 \mid d, \gamma}^{\mathrm{a}}(s)$ & $4 \exp \left(d^{2} \gamma\left(s^{2}-s\right)\right)$ \\
\hline & $\Phi_{\Lambda, 5 \mid d, \bar{\gamma}, m}^{\mathrm{a}}(s)$ & $4\left(\frac{m}{m-d^{2} \bar{\gamma}\left(s^{2}-s\right)}\right)^{m}$ \\
\hline \multirow{3}{*}{ Case 6} & $f_{\Lambda, 6 \mid d, \gamma}^{\mathrm{a}}(\lambda)$ & $2 \mathcal{N}_{d^{2} \gamma, 2 d^{2} \gamma}(\lambda)$ \\
\hline & $\Phi_{\Lambda, 6 \mid d, \gamma}^{\mathrm{a}}(s)$ & $2 \exp \left(d^{2} \gamma\left(s^{2}-s\right)\right)$ \\
\hline & $\Phi_{\Lambda, 6 \mid d, \bar{\gamma}, m}^{\mathrm{a}}(s)$ & $2\left(\frac{m}{m-d^{2} \bar{\gamma}\left(s^{2}-s\right)}\right)^{m}$ \\
\hline
\end{tabular}




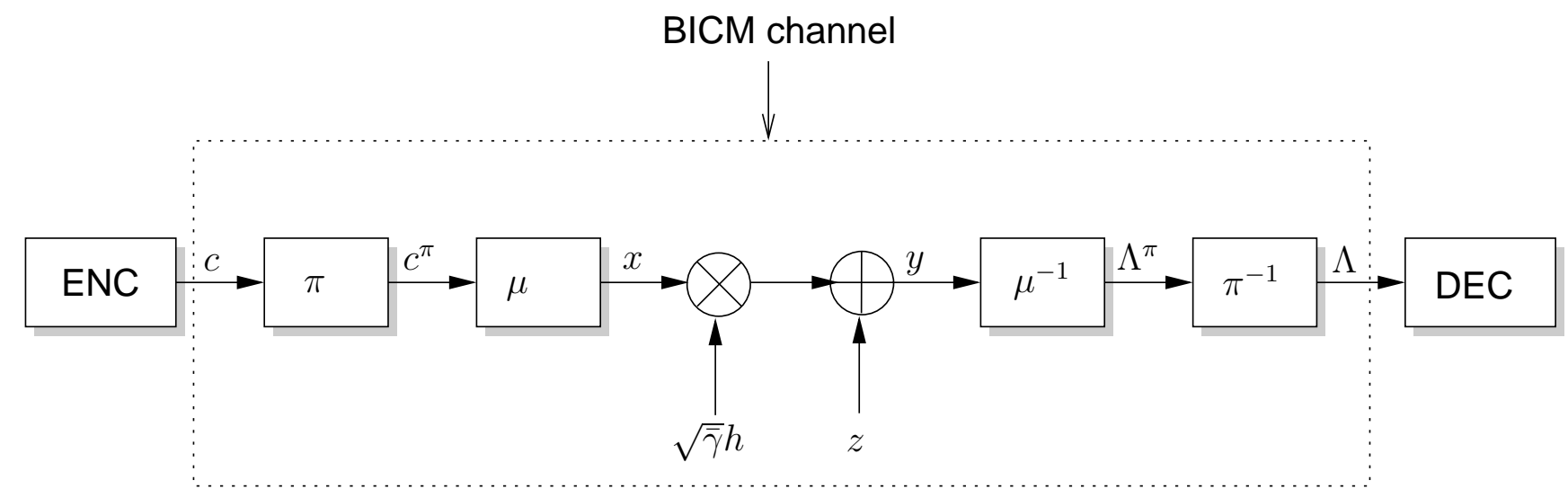

Fig. 1. Block diagram of BICM transmission over a fading AWGN channel. Also indicated is the binary-input continuous-output equivalent BICM channel. 


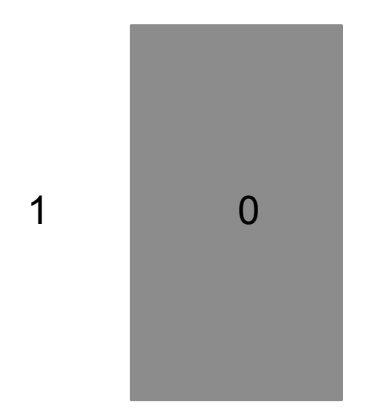

Case I

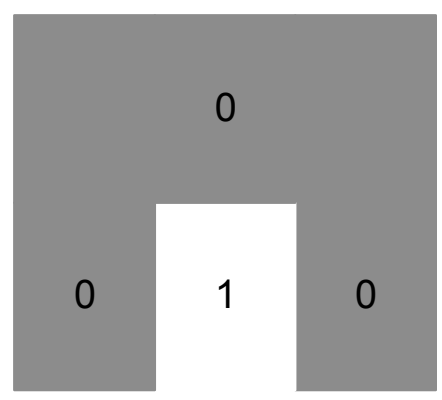

Case IV

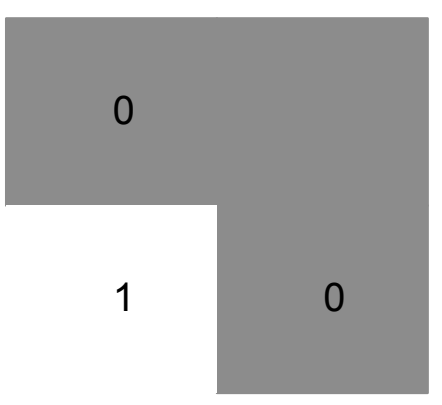

Case II

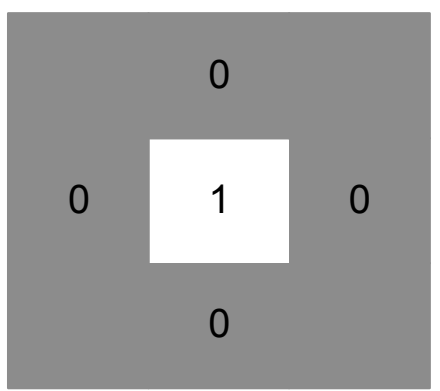

Case V

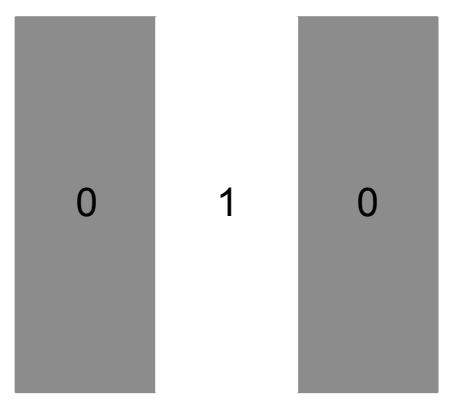

Case III

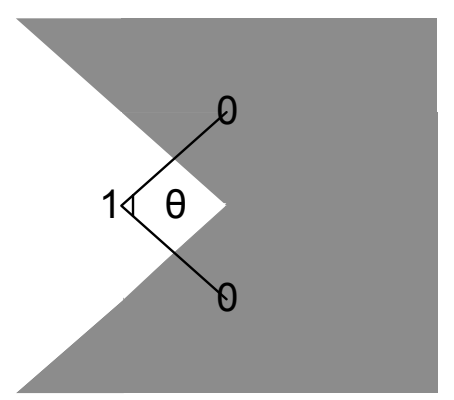

Case VI

Fig. 2. Illustration for possible sets of nearest competitive signal points $\mathcal{A}_{j, x}$ for general QAM and PSK constellations. The " 1 " represents the transmitted signal point $x$, and the " 0 " show the elements of $\mathcal{A}_{j, x}$. The shaded areas indicates $\mathbb{D}(\lambda \mid j, x, \gamma)$ for $\lambda=0$. For $\lambda>0$ the boundaries move towards $x$, for $\lambda<0$ the boundaries move towards the competitive signal points. 


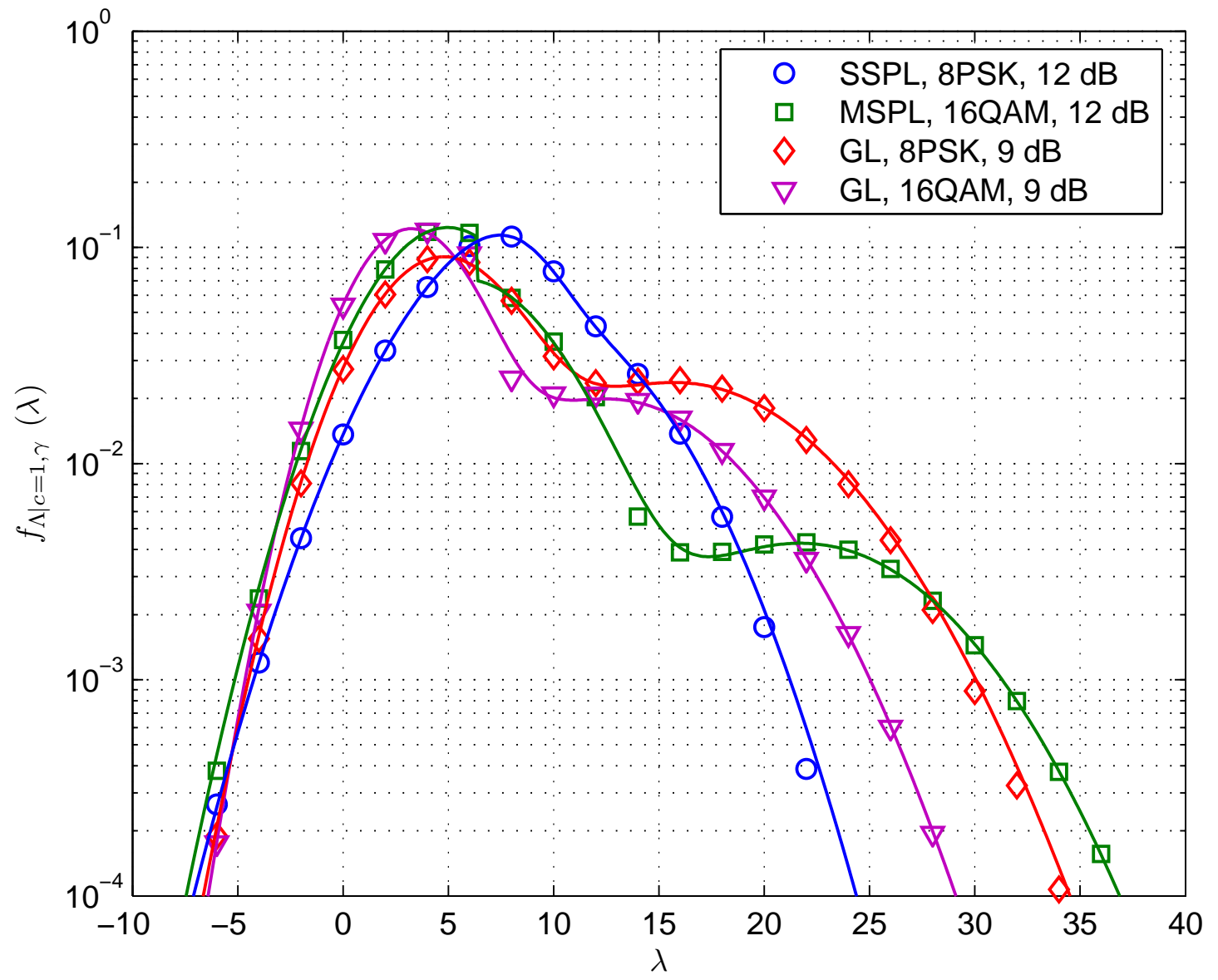

Fig. 3. Probability density functions of reliability metrics for BICM transmission over the nonfading AWGN channel for different constellations and labeling. Lines represent the PDF approximation given in (20) and (21) while markers represent the estimated histograms through simulative measurement. 


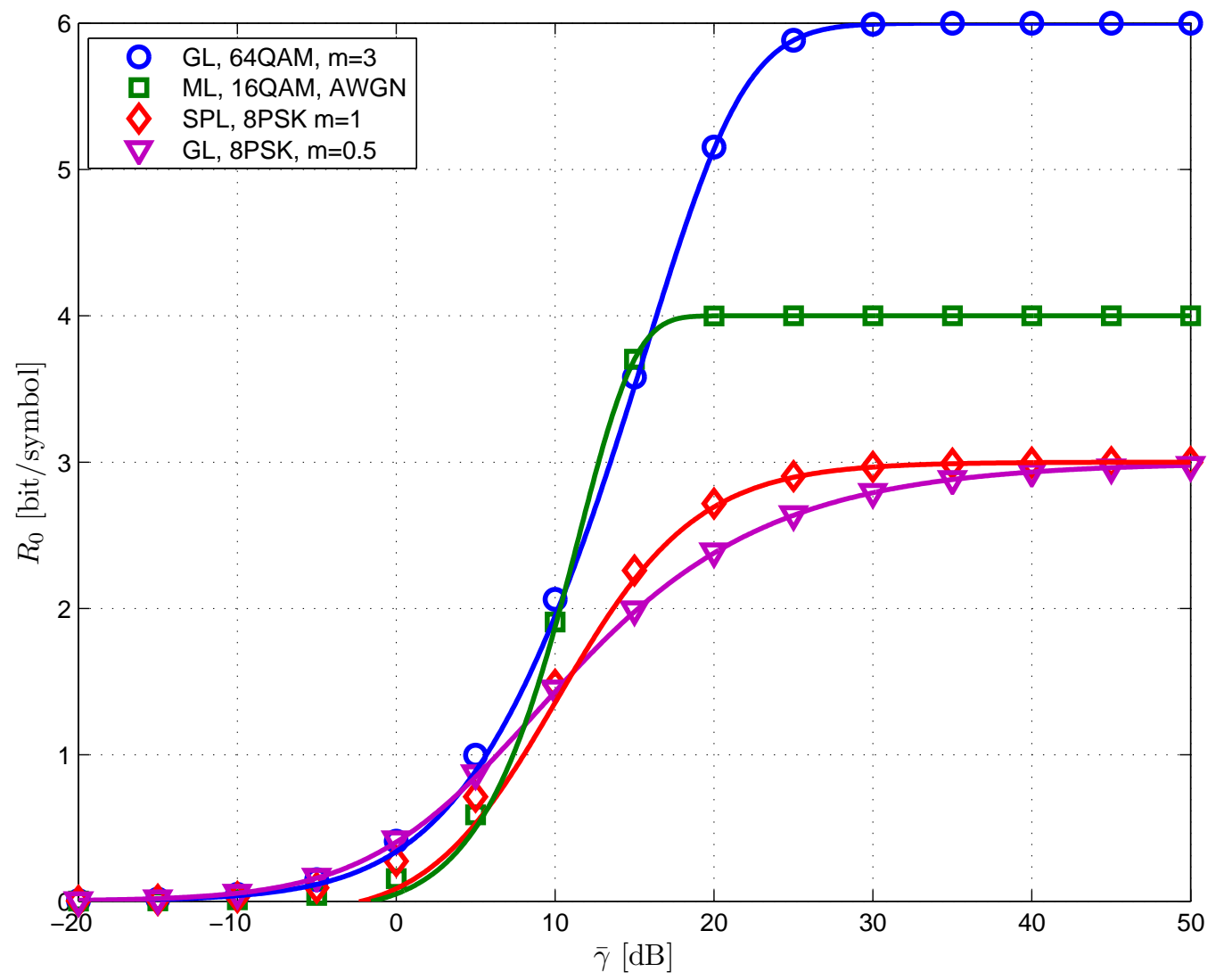

Fig. 4. Cutoff Rate $R_{0}$ for BICM channel with different constellations, labeling rules, and channels. Lines are obtained from evaluation of (12) using the approximation for the Laplace transform derived in Section III-B, while markers are obtained from Monte Carlo simulation. 


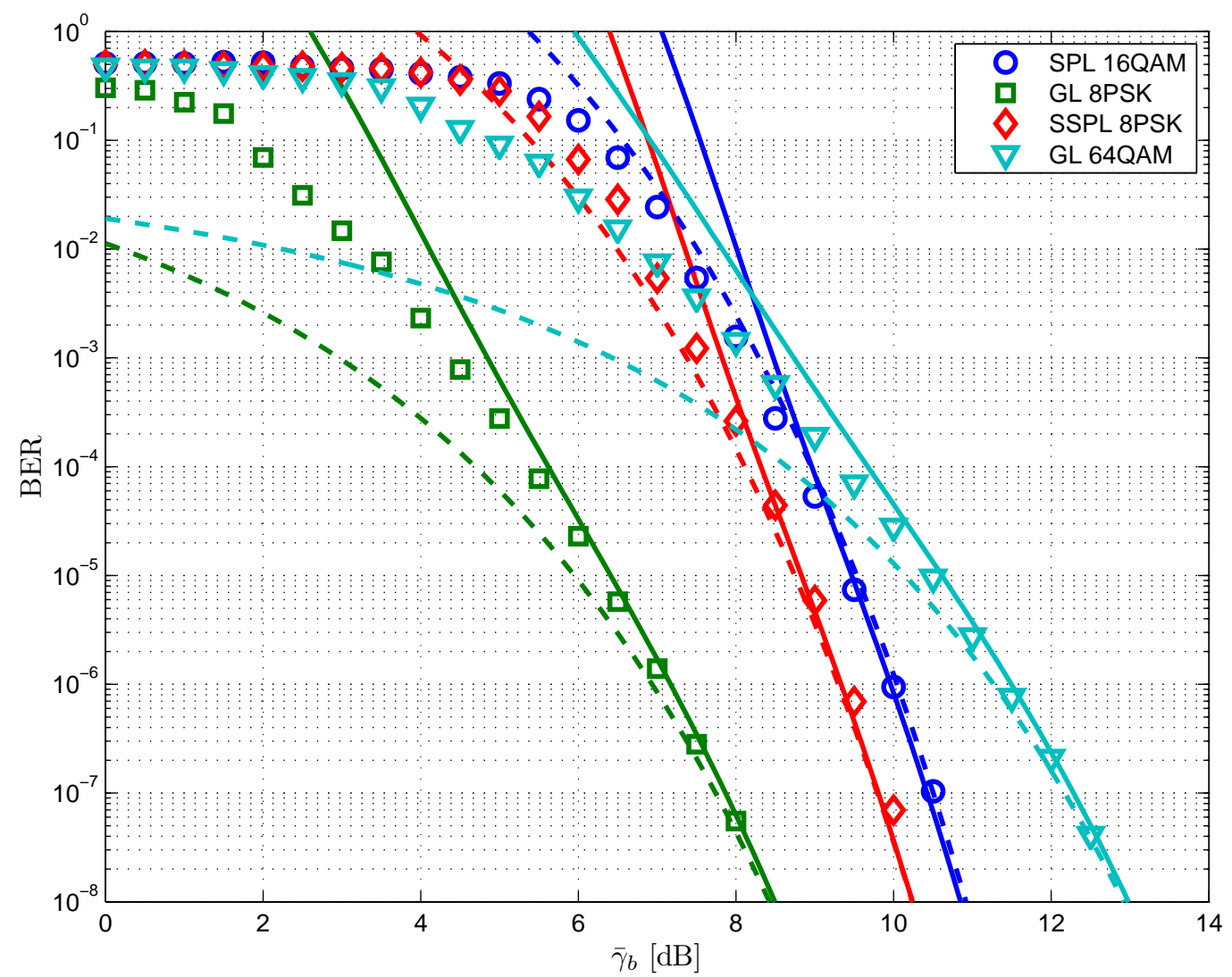

Fig. 5. BER of BICM transmission over nonfading AWGN channel for a 64-state convolutional code of rate 1/2. Solid lines: BER union bound. Dashed lines: Asymptotic analysis with (42). Markers: Simulation results 


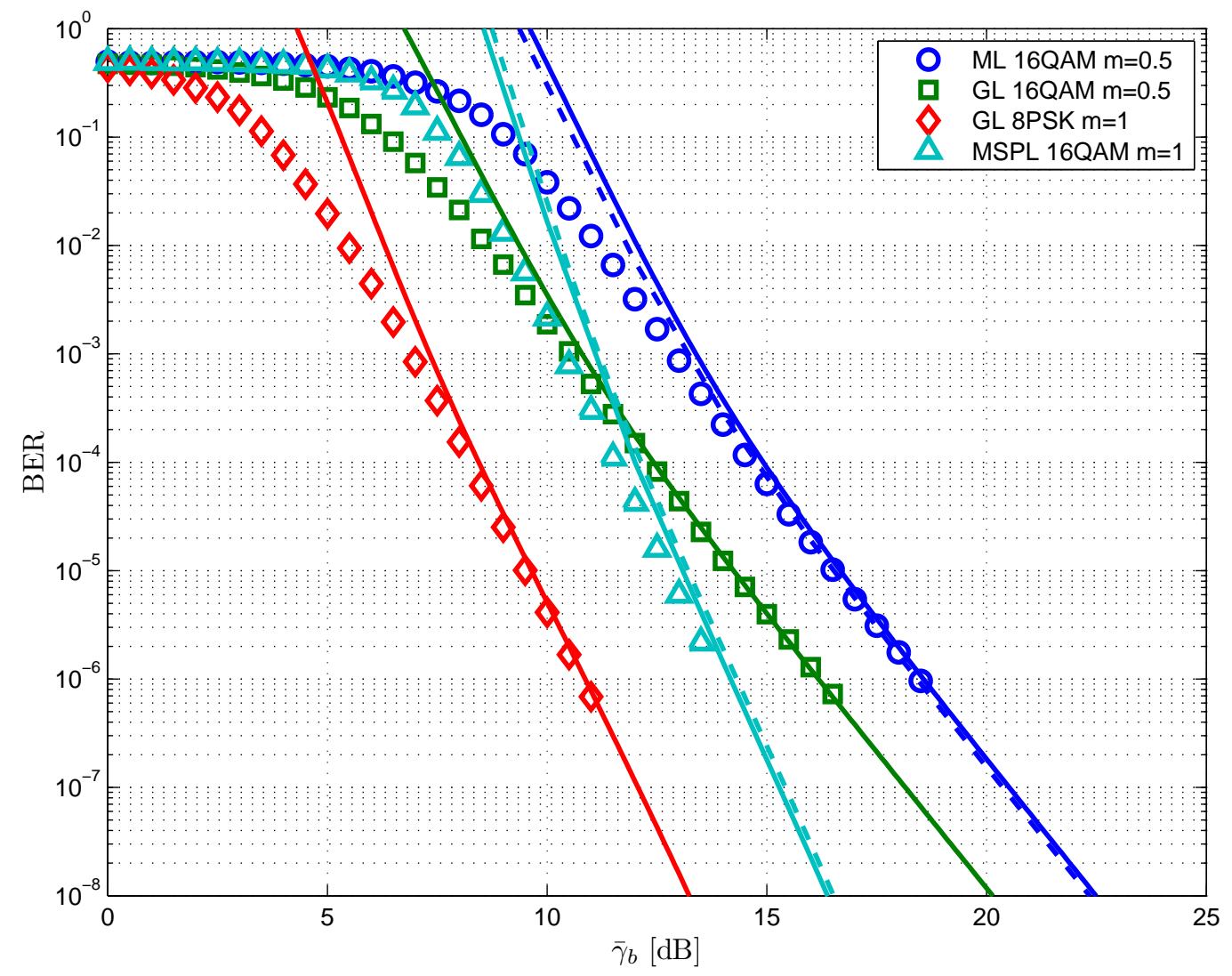

Fig. 6. BER of BICM transmission over Nakagami- $m$ fading channel for a 64 -state convolutional code of rate $1 / 2$. Solid lines: BER union bound using the exact closed-form solutions for integrals (28), (29). Dashed lines: BER union bound using the approximations (62), (67). Markers: Simulation results 


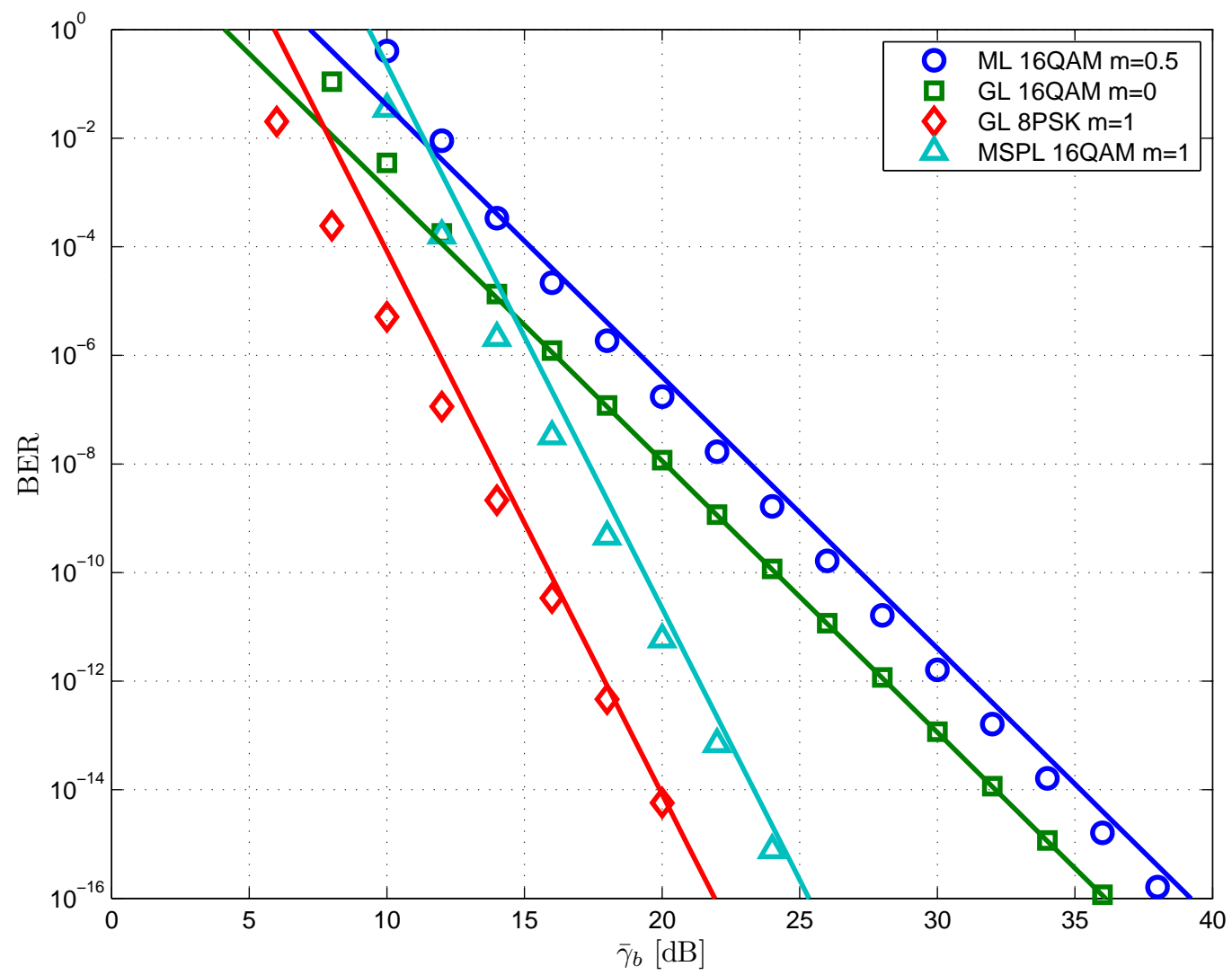

Fig. 7. BER of BICM transmission over Nakagami- $m$ fading channel for a 64 -state convolutional code of rate $1 / 2$. Solid lines: Asymptotic analysis with (48). Markers: BER union bound. 

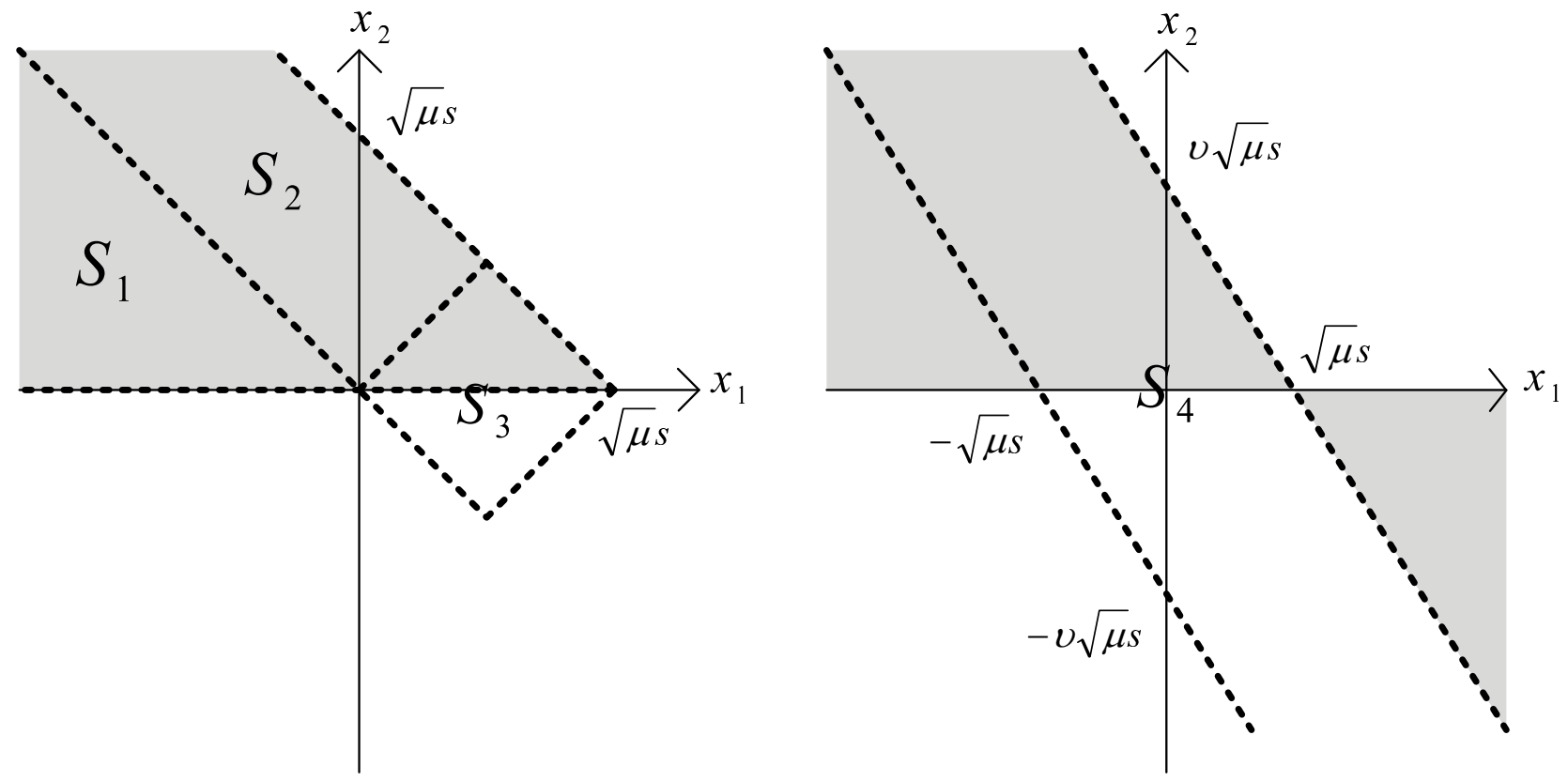

Fig. 8. The graphical representation of integrals (51) (left) and (54) (right). Shaded areas are integration supports, and dashed lines indicate decomposition into support areas for which the integrals can be solved. 